\title{
Toxoplasma gondii protease TgSUB1 is required for cell surface processing of micronemal adhesive complexes and efficient adhesion of tachyzoites
}

\author{
Vanessa Lagal, ${ }^{1,2 \dagger}$ Emily M. Binder, ${ }^{1,2}$ \\ My-Hang Huynh, ${ }^{3}$ Bjorn F. C. Kafsack, ${ }^{3 \ddagger}$ \\ Philippa K. Harris, ${ }^{1,2}$ Roberto Diez, ${ }^{4}$ Dawn Chen, ${ }^{4}$ \\ Robert N. Cole, ${ }^{4}$ Vern B. Carruthers ${ }^{3}$ and \\ Kami Kim ${ }^{1,2 *}$ \\ Departments of ${ }^{1}$ Medicine and ${ }^{2}$ Microbiology \& \\ Immunology, Albert Einstein College of Medicine, Bronx, \\ NY 10461, USA. \\ ${ }^{3}$ Department of Microbiology and Immunology, \\ University of Michigan, Ann Arbor, MI 48109, USA. \\ ${ }^{4}$ Mass Spectrometry and Proteomics Facility, Institute for \\ Basic Biomedical Sciences, Johns Hopkins School of \\ Medicine, Baltimore, MD 21205, USA.
}

\section{Summary}

Host cell invasion by Toxoplasma gondii is critically dependent upon adhesive proteins secreted from the micronemes. Proteolytic trimming of microneme contents occurs rapidly after their secretion onto the parasite surface and is proposed to regulate adhesive complex activation to enhance binding to host cell receptors. However, the proteases responsible and their exact function are still unknown. In this report, we show that $T$. gondii tachyzoites lacking the microneme subtilisin protease TgSUB1 have a profound defect in surface processing of secreted microneme proteins. Notably parasites lack protease activity responsible for proteolytic trimming of MIC2, MIC4 and M2AP after release onto the parasite surface. Although complementation with fulllength TgSUB1 restores processing, complementation of $\Delta s u b 1$ parasites with TgSUB1 lacking the GPI anchor ( $\triangle$ sub1:: $\triangle$ GPISUB1) only partially restores microneme protein processing. Loss of TgSUB1 decreases cell attachment and in vitro

Received 18 February, 2010; revised 21 June, 2010; accepted 9 July, 2010. ${ }^{*} F o r$ correspondence. E-mail: kami.kim @ einstein.yu.edu; Tel. (+1) 718430 2611; Fax (+1) 7184308968.

Present addresses: 'Institut Cochin, INSERM U-1016, CNRS UMR8104, Paris, France; ‡Lewis-Sigler Institute for Integrative Genomics, Princeton University, Princeton, NJ 08544, USA. gliding efficiency leading to lower initial rates of invasion. $\Delta$ sub1 and $\triangle$ sub1:: $\triangle$ GPISUB1 parasites are also less virulent in mice. Thus TgSUB1 is involved in micronemal protein processing and regulation of adhesive properties of macromolecular adhesive complexes involved in host cell invasion.

\section{Introduction}

The apicomplexan phylum of protozoa contains numerous parasites of veterinary (Cryptosporidium, Neospora, Eimeria) and medical (Plasmodium, Cryptosporidium, Toxoplasma) importance. The parasite Toxoplasma gondii causes severe clinical diseases in immunocompromised patients and, when contracted during pregnancy, can lead to birth defects or abnormal development. Apicomplexan parasites share a common substrate dependent locomotion termed gliding motility used for active host cell penetration and tissue migration (Carruthers and Sibley, 1997; Dubremetz et al., 1998; Carruthers et al., 1999a). Secretion of apical organelles called micronemes and rhoptries leads to the formation of an intimate binding interface junction connecting host cell receptors and parasite adhesive proteins. Host cell invasion relies on the translocation of transmembrane adhesive proteins that form a bridge between the host cell and the parasite actomyosin motor which provides motive force for active penetration (Dobrowolski and Sibley, 1996; Meissner et al., 2002b; Keeley and Soldati, 2004; Baum et al., 2006).

Microneme proteins (MIC) contain domains with homology to adhesive motifs from higher eukaryotes that are thought to be involved in the binding to host cell receptors. Several MICs bind carbohydrate receptors on host cells (Fourmaux et al., 1996; Garcia-Réguet et al., 2000; Brecht et al., 2001; Cerede et al., 2002; Harper et al., 2004; Barragan etal., 2005; Saouros et al., 2005; Friedrich et al., 2010). Genetic studies with MIC knock-out mutants have confirmed a significant role for MICs in attachment, invasion and virulence (Huynh et al., 2003; Cerede et al., 2005; Huynh and Carruthers, 2006). MIC proteins are assembled into macromolecular adhesive 
complexes during transit through the secretory pathway (Reiss et al., 2001; Meissner et al., 2002a; Jewett and Sibley, 2004) and are extensively processed during their intracellular trafficking and after release onto the parasite surface (Dowse and Soldati, 2004; Carruthers, 2006). Proteolytic processing of microneme proteins after release onto the parasite surface is essential for successful completion of host cell invasion (Conseil et al., 1999; Brossier et al., 2003; Harper et al., 2006; Teo et al., 2007). Three protease activities have been described on the parasite surface, termed Microneme Protein Protease 1-3 (MPP1, MPP2, MPP3) (Zhou et al., 2004). MPP1 is responsible for intramembranous cleavage of MIC proteins within their transmembrane domains, resulting in shedding of adhesive complexes from the cell surface (Brossier et al., 2005; Dowse et al., 2005). MPP2 and MPP3 are involved in the proteolytic trimming of several components of microneme adhesive complexes including MIC2, M2AP and MIC4. MPP2 and MPP3 are distinguished by their differential susceptibility to ALLN and other protease inhibitors, but their molecular nature has not been identified (Carruthers et al., 2000; Brecht et al., 2001; Zhou et al., 2004). It has been speculated that the surface proteolytic trimming activities of MPP2 and MPP3 are involved in the exposure of adhesive domains of MICs required for tight binding to host cell receptors (Carruthers and Blackman, 2005).

Here we elucidate the role of the $T$. gondii subtilisin-like serine protease TgSUB1 in MIC surface proteolytic trimming and show TgSUB1 is required for MPP2 and MPP3 activities. TgSUB1 is a GPI-anchored micronemal protease that is first released onto the surface of parasites during invasion and then shed into the media with other micronemal proteins (Miller et al., 2001; Binder et al., 2008). Because of its microneme and surface localization, we hypothesized that TgSUB1 mediates MIC protein processing during host cell invasion. We show that TgSUB1 is involved in the surface processing of several MICs including the adhesive complex M2AP-MIC2 and the protein MIC4. Loss of TgSUB1-dependent MIC surface processing affects parasite cell attachment, host cell invasion, as well as in vitro gliding efficiency. Moreover, parasites lacking TgSUB1 have a defect in virulence leading to a delay in time death in the mouse model.

\section{Results}

Tachyzoites lacking TgSUB1 have altered microneme protein processing

The TgSUB1 gene was disrupted by homologous recombination in two independent cell lines: RH GFP- $\Delta h x g p r t$ and $\mathrm{RH} \Delta h x g$ prt background as described elsewhere (Binder et al., 2008). Loss of TgSUB1 expression was confirmed by quantitative real-time PCR, Western blot and immunofluorescence analyses (Fig. 1A and Fig. S1). The expression, targeting and intracellular processing of MICs AMA1, MIC2, MIC3, MIC4, MIC5, MIC6 and M2AP were not affected in the $\Delta s u b 1$ strain (Fig. 1C; Binder et al., 2008; and data not shown). To determine if MIC proteolytic processing on the parasite surface was impaired, secretion of microneme proteins MIC2, MIC4 and M2AP, known substrates of MPP2 and MPP3 was analysed by Western blot. Efficiency of the secretion procedure was confirmed by Western blot analysis to show enhancement of secreted forms and absence of cytosolic proteins (data not shown). M2AP is first cleaved in its C-terminal region by MPP3 generating the fragment M2AP-1 and further cleaved by MPP2 generating three additional fragments (M2AP 2-4, Fig. 1B) (Zhou et al., 2004). MIC4 (72 kDa) is cleaved within its N-terminus to generate a $70 \mathrm{kDa}$ species and is further processed by MPP2 in its C-terminus to generate 50 and $15 \mathrm{kDa}$ products (Carruthers et al., 2000; Brecht et al., 2001; Zhou et al., 2004). MIC4 $50 \mathrm{kDa}$ and M2AP 1-4 secreted products are no longer detected in the $\Delta s u b 1$ strain and this loss of processed forms is associated with accumulation of the M2AP mature form (Fig. 1C). Results were similar in both $\Delta s u b 1$ strains (data not shown). In wild-type parasites, MIC2 is cleaved in its N-terminus by MPP2 removing a $5 \mathrm{kDa}$ fragment (Fig. 1B) (Carruthers et al., 2000; Zhou et al., 2004), but in the $\Delta s u b 1$ strain, the MIC2secreted product was of higher molecular weight, indicating that it too was no longer processed (Fig. 1C).

To identify other potential substrates for TgSUB1, we performed a global proteomic analysis of the ESA (excreted-secreted antigen) profile of $\Delta s u b 1$ (GFP background) and its sibling transfectant Clone 2 (which continues to express TgSUB1). ESA preparations were analysed by two-dimensional differential gel electrophoresis (2D-DIGE) analysis (Fig. 2A). Proteins that were diminished or absent in the $\Delta$ sub1 strain are visible as green spots. As expected, TgSUB1-secreted products were not observed in the $\Delta$ sub1 strain (80 kDa, $70 \mathrm{kDa}$, $40 \mathrm{kDa}$ products and TgSUB1 prodomain spot D8) (Fig. 2A, Fig. S2 and Table 1). Inhibition of MIC2, MIC4 and M2AP processing was confirmed as depicted by the diminution of the MIC2 $95-90 \mathrm{kDa}$ products, M2AP 1-4 products and the MIC4 50 and $15 \mathrm{kDa}$ products (spots D3-D4-D21, Fig. 2A, Fig. S2 and Table 1). Moreover, 2D-DIGE analysis confirmed the accumulation of the M2AP mature form and revealed the absence of MIC4 $70 \mathrm{kDa}$ product and accumulation of the MIC4 $72 \mathrm{kDa}$ (spot U2) precursor in the $\Delta$ sub1 (Fig. 2A, Table 1 and Fig. S2).

Several additional proteins with differences in abundance were identified by mass spectrometry (Table 1). Among these was perforin like protein 1 (PLP1) which 

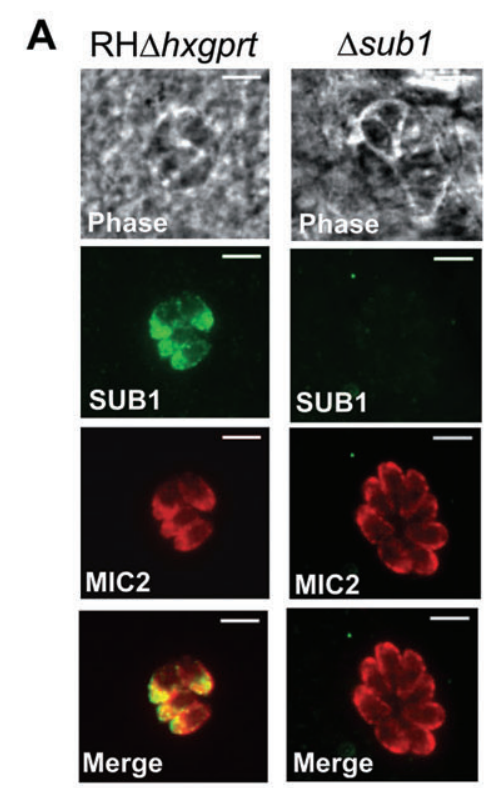

Clone 2
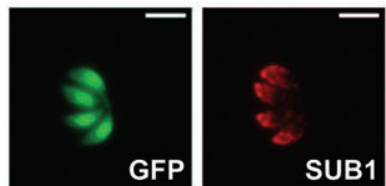

C

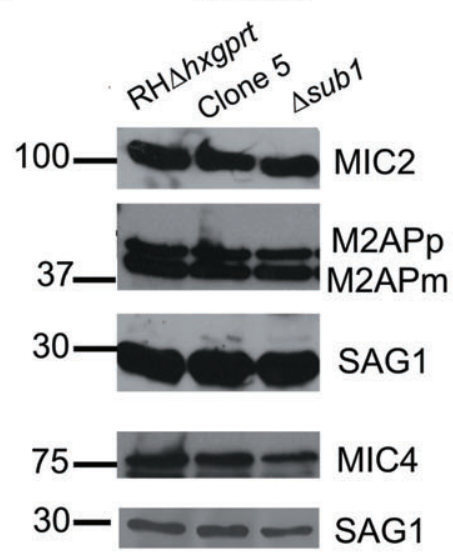

Clone 5
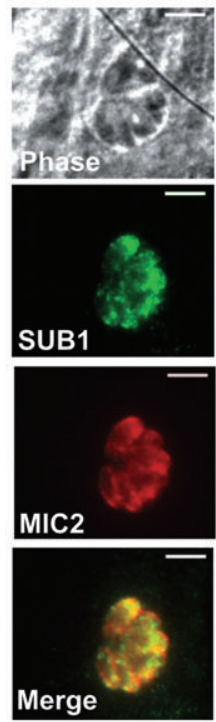

$\triangle$ sub1GFP
B

Pre-secretion

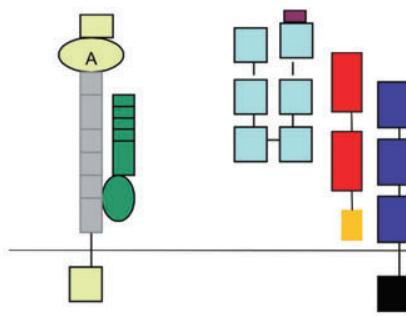

MIC2-M2AP
EGF-like domain

TSP-like domain Apple-like domain

Galectin-like domain

Microneme Adhesive Repeat

Microneme discharge

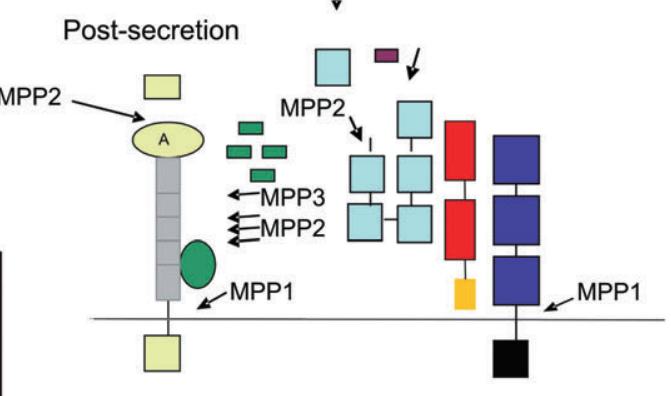

Secreted

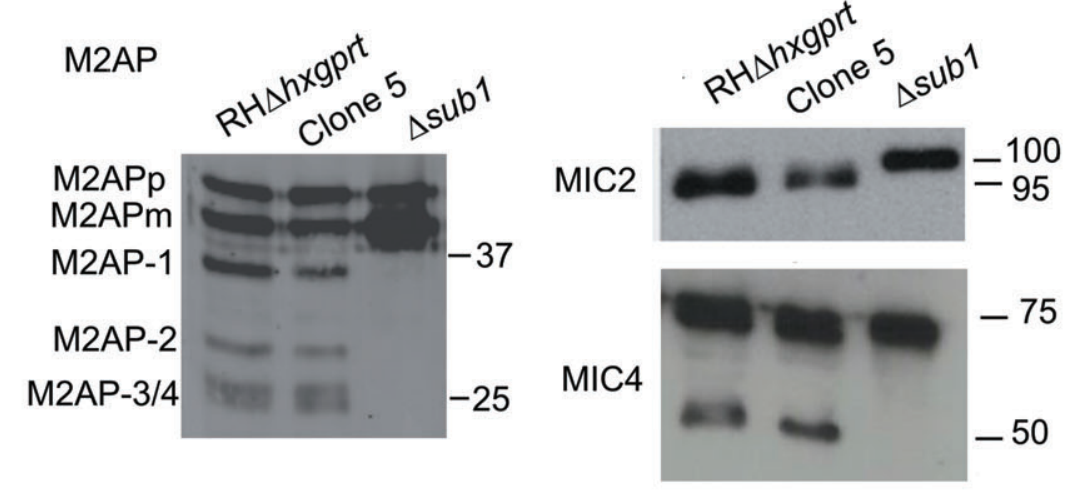

Fig. 1. TgSUB1 disruption alters MIC proteolytic processing.

A. Immunofluorescence of $\Delta s u b 1$ strains and sibling clones (Clone 2 for $\Delta s u b 1 \mathrm{GFP}$; Clone 5 for $\Delta s u b 1$ ). Controls include the wild-type RH GFP- $\Delta h x g p r t$ and $\mathrm{RH} \Delta h x g p r t$ strains. Siblings correspond to wild-type parasites stably transfected with the selectable marker but without TgSUB1 disruption. TgSUB1 is labelled with the TgSUB1 AE653 rabbit antiserum. MIC2 is labelled with the mouse mAb 6D10 and was used as a marker for microneme localization. Scale bar is $5 \mu \mathrm{m}$.

B. Schematic representation of the cell surface proteolytic trimming of the micronemal adhesive complexes MIC2-M2AP and MIC1-4-6. ' $A$ ' denotes the A-domain of MIC2 protein. Microneme Adhesive Repeat refers to the specialized MIC1 domain which discriminates between glycan residues (Blumenschein et al., 2007). Activity of the proteases MPP1, 2 and 3 is represented. Because the first step in M2AP processing is not inhibited by protease inhibitors that inhibit MPP2 (ALLM, ALLN), a second protease activity termed MPP3 was proposed (Zhou et al., 2004). The MPP1 MIC cleavage sites are consistent with MPP1 being a rhomboid serine protease such as the parasite surface rhomboids TgROM4 or TgROM5 (Brossier et al., 2005; Dowse et al., 2005; Buguliskis et al., 2010).

C. Western blot analysis of the MIC2-4 and M2AP cellular and secreted products. Blots were probed with appropriate antibodies as described in Experimental procedures. Molecular standards are in $\mathrm{kDa}$. The rabbit anti-MIC4 antibody used was raised against the A1 and A2 apple domains, and recognizes the 72 and $70 \mathrm{kDa}$ precursor forms and the $50 \mathrm{kDa}$ secreted product but not the $15 \mathrm{kDa}$ secreted product. M2APp: precursor form; M2APm: mature form. 


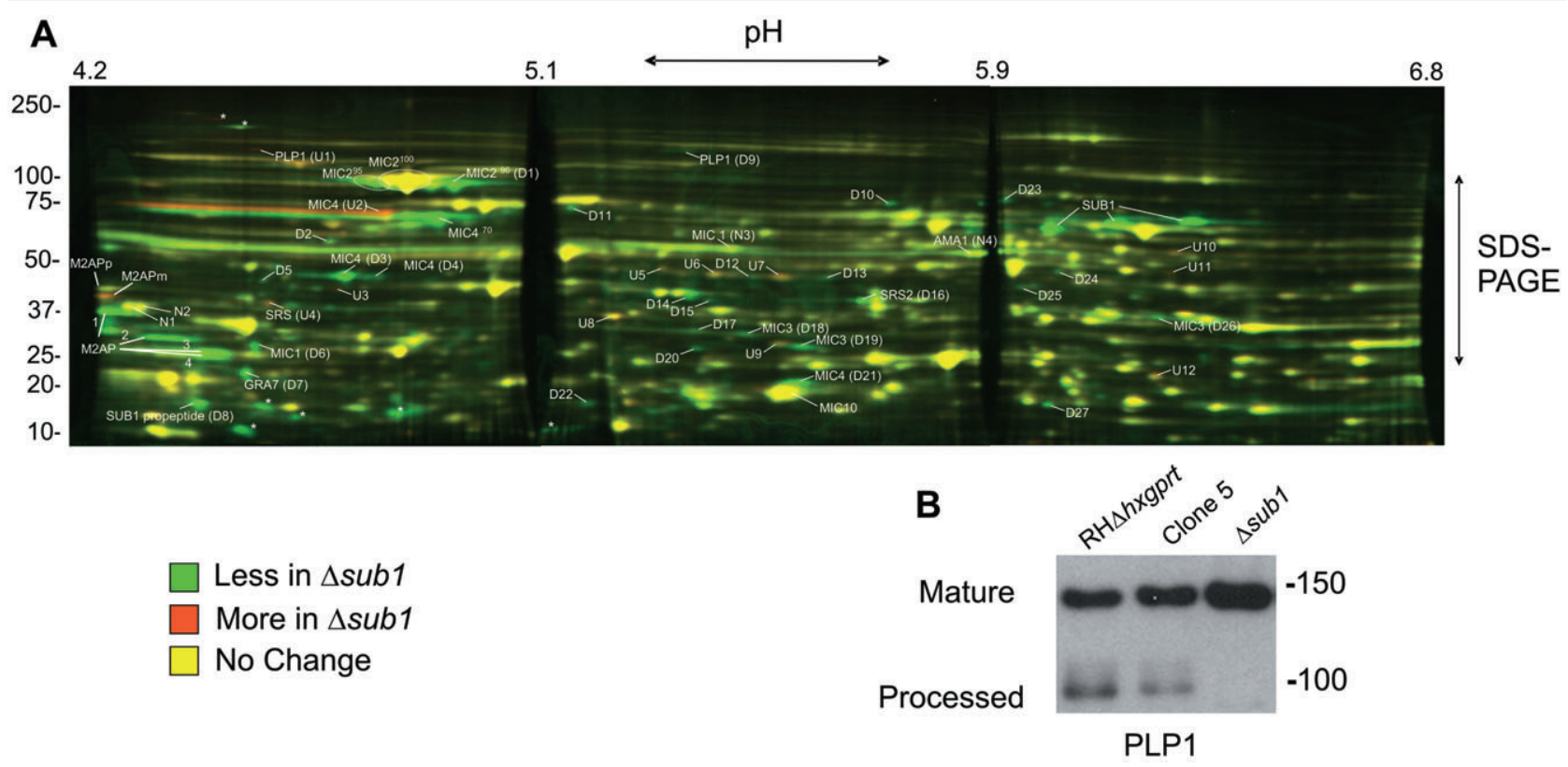

Fig. 2. 2D-DIGE analysis of microneme-secreted products.

A. Secreted products of the $\triangle$ sub1GFP and Clone 2 strains were covalently labelled with Cy3 (green) or Cy5 (red), respectively, and separated by a two-dimensional gel electrophoresis ( $\mathrm{pH} 4.2-6.8$ first dimension and 12.5\% SDS-PAGE second dimension). Protein spots that are diminished or absent in the $\Delta$ sub1-secreted sample compared with the Clone 2 (a sibling transfectant that still expresses TgSUB1) are depicted as green spots while proteins that are increased are depicted as orange spots. Yellow spots depict proteins that do not change significantly in abundance between the two strains. Marked proteins were excised and identified by mass spectrometry analysis. The asterisks indicate spots not sufficiently visible on the Coomassie gel to excise.

B. Western blot analysis of the PLP1-secreted products. PLP1 was detected with appropriate antibody. Molecular weights are in kDa.

Table 1. Summary of proteins with a signal peptide identified by 2D-DIGE and mass spectrometry.

\begin{tabular}{|c|c|c|c|c|c|}
\hline Spot & Protein & $\begin{array}{l}\text { Gene ID Release } 4.3 \\
\text { http://www.toxodb.org }\end{array}$ & Gene ID Release 5 & $\begin{array}{l}\mathrm{MW} \\
\mathrm{kDa}\end{array}$ & $\mathrm{pl}$ \\
\hline U1 & PLP1 & 20.m03849 & TGGT1_016570 & 120 & 4.5 \\
\hline U2 & MIC4 & 25.m00006 & TGGT1_021130 & 63 & 5.1 \\
\hline U4 & SRS & 641.m03153 & TGGT1_121850 & 37 & 4.5 \\
\hline N1 & Ca binding & 44.m00001 & TGGT1_118190 & 35 & 4.3 \\
\hline $\mathrm{N} 2$ & Ca binding & 44. m00001 & TGGT1_118190 & 35 & 4.3 \\
\hline N3 & MIC1 & 80.m00012 & TGGT1_031650 & 60 & 5.5 \\
\hline N4 & AMA1 & 55.m00005 & TGGT1_076680 & 60 & 5.9 \\
\hline D1 & MIC2 & 20.m00002 & TGGT1_019450 & 90 & 4.9 \\
\hline D3 & MIC4 & $25 . \mathrm{m} 00006$ & TGGT1_021130 & 63 & 5.1 \\
\hline D4 & MIC4 & 25.m00006 & TGGT1_021130 & 63 & 5.1 \\
\hline D6 & MIC1 & 80.m00012 & TGGT1_031650 & 28 & 4.5 \\
\hline D7 & GRA7 & 20.m00005 & TGGT1_017550 & 22 & 4.5 \\
\hline D8 & SUB1 propeptide & 20.m00387 & TGGT1_016650 & 16 & 4.4 \\
\hline D9 & PLP1 & 20.m03849 & TGGT1_016570 & 115 & 5.4 \\
\hline D10 & SUB1 & 20.m00387 & TGGT1_016650 & 82 & 5.1 \\
\hline D16 & SRS2 & 44. $\mathrm{m} 00010$ & TGGT1_113990 & 39 & 7 \\
\hline D18 & MIC3 & 641.m00002 & TGGT1_121730 & 35 & 5.5 \\
\hline D19 & MIC3 & 641.m00002 & TGGT1_121730 & 30 & 5.6 \\
\hline D21 & MIC4 & 25.m00006 & TGGT1_021130 & 20 & 5.6 \\
\hline D22 & Hypothetic protein & 31.m00928 & TGGT1_039470 & 17 & 5.2 \\
\hline D23 & SUB1 & 20.m00387 & TGGT1_016650 & 82 & 5.1 \\
\hline D26 & MIC3 & 641.m00002 & TGGT1_121730 & 38 & 6.1 \\
\hline
\end{tabular}

Spots of interest were excised and proteins were identified by mass spectrometry analysis. Proteins were identified by searching the data against the EPICDB database (http://toro.aecom.yu.edu/biodefense/) using MASCOT and are based on two or more peptide sequences with scores above the $95 \%$ confidence threshold. $U$ denotes a protein whose abundance is higher (up) in the $\Delta$ sub1 strain; $N$ a protein whose abundance is unchanged (neutral) in the $\Delta$ sub1 strain and D a protein less abundant (down) in the $\Delta$ sub1 strain. MW, molecular weight; pl, isoelectric point; SRS, SAG-related sequence; Ca, calcium. Proteins were identified using ToxoDB.org Release 4 gene predictions. Release 5 gene identification numbers for GT-1, a type I strain like RH, are also shown.

(C) 2010 Blackwell Publishing Ltd, Cellular Microbiology, 12, 1792-1808 
was originally identified in a proteomic screen of secretory products (Zhou et al., 2004). 2D-DIGE analysis revealed accumulation of a precursor form and diminution of a probable processed form (spots U1 and D9; Fig. 2A and Table 1). This was confirmed by Western blot analysis, which showed the absence of PLP1 processed product in the $\Delta s u b 1$ strain (Fig. 2B).

The abundance of several other proteins in ESA was changed in the $\Delta$ sub1 strain including the microneme proteins MIC1 and MIC3 (Table 1, Fig. 2A). In addition to known micronemal proteins, other secretory proteins with altered abundance including the dense granule protein GRA7 and two SAG-related sequence (SRS) proteins (TGGT1_121850 and TGGT_113990; Table 1, Fig. 2A, spots D7-D16-U4). Previous studies characterizing ESA components have typically included dense granule proteins and surface antigens (Cesbron-Delauw et al., 1989; Zhou et al., 2004). The differences in ESA profile were reproducible in three independent biological replicates analysed by 2D-DIGE, as well as numerous independent ESA secretion analyses of both $\Delta s u b 1$ strains.

\section{TgSUB1 GPI anchorage is necessary for MPP2 activity}

The $\Delta s u b 1$ strain was complemented with full-length TgSUB1 ( $\triangle$ sub1::FLSUB1) or a TgSUB1 lacking the C-terminal GPI signal sequence ( $\triangle$ sub1:: $\triangle$ GPISUB1). A clone where only the CAT cassette has been integrated was also selected as a control ( $\triangle$ sub1pCAT). TgSUB1 complementation and CAT cassette integration were confirmed by Southern blot (Fig. S1B). TgSUB1 expression was restored to wild-type levels as shown by quantitative real-time PCR and Western blot analyses (Fig. S1C and D). Both FLSUB1 and $\triangle$ GPISUB1 proteins were correctly targeted to the micronemes consistent with prior results showing the GPI anchor is not involved in TgSUB1 microneme targeting (Fig. 3A; Binder et al., 2008). Upon microneme discharge, TgSUB1 was secreted and correctly processed (Fig. S1E).

We performed IFA on extracellular parasites stimulated for microneme secretion and on invading parasites to test if TgSUB1 protein deposition upon the parasite surface after microneme secretion is dependent on the GPI anchor. In both the $\triangle$ GPISUB1- and FLSUB1complemented strains, TgSUB1 was detected on the parasite surface, as observed for many MIC proteins lacking membrane association such as MIC5 (Brydges et al., 2006) and M2AP (Rabenau et al., 2001; Huynh et al., 2003). The expression, targeting and intracellular processing of the rhoptry and microneme proteins MIC2, MIC3, MIC4, MIC5, MIC6, M2AP and ROP 2,3,4 were not affected in the $\Delta$ sub1-complemented strains (Fig. $3 C$, Fig. S1F and data not shown).

Complementation of the MIC protein surface processing was investigated by Western blot analysis of ESA from the complemented strains. Processing of MIC2, M2AP and MIC4 was restored in $\triangle$ sub1::FLSUB1consistent with TgSUB1 being required for the proteolytic activity responsible for cell surface trimming of these proteins (Fig. 3C). Unexpectedly, only the first processing step of M2AP, which is ascribed to MPP3, was restored in the $\triangle s u b 1:: \triangle$ GPISUB1 strain suggesting that SUB1 GPI surface anchorage is necessary for restoration of full proteolytic activity, including cleavage of substrates cleaved by MPP2.

\section{Loss of TgSUB1 impairs tachyzoite attachment and invasion efficiency}

Since surface proteolytic trimming is proposed to be involved in the activation of MICs for host cell interaction, we tested the ability of the different $\Delta s u b 1$ strains to attach to fixed host cells. A significant decrease in attachment (30-40\%) was observed for $\Delta s u b 1$, $\triangle$ sub $1:: \triangle$ GPISUB1 and $\triangle$ sub1pCAT strains (Fig. 4A). The ability of the different strains to invade human foreskin fibroblasts was assessed by a Red/Green invasion assay, which discriminates between parasites that can attach versus those that have successfully invaded a host cell (Huynh et al., 2003). After a 15 min incubation, invasion of $\triangle s u b 1, \Delta s u b 1:: \Delta$ GPISUB1 and $\triangle$ sub 1p CAT strains was decreased by $45-60 \%$ compared with $\mathrm{RH} \Delta h x g p r t$ and Clone 5 (Fig. 4B).

Fig. 3. Complementation of the MIC surface processing by TgSUB1-complemented $\Delta$ sub1 strains.

A. Immunofluorescence of TgSUB1-complemented $\Delta$ sub1 strains. All strains were simultaneously labelled with TgSUB1 antiserum AE653 and MIC2 monoclonal antibody 6D10, a marker for micronemes. Scale bar is $5 \mu \mathrm{m}$.

B. Surface detection of TgSUB1 in the three representative strains: $\triangle$ sub1::FLSUB1, $\triangle$ sub1:: $\triangle$ GPISUB1 and $\triangle$ sub1. Extracellular parasites were treated with the ionophore A23187 to induce microneme discharge. Tachyzoite nuclei were stained with DAPI and TgSUB1 was immunolabelled with the TgSUB1-specific antibody AE653 without cellular permeabilization. Freshly egressed parasites were inoculated onto HFF monolayers and invading tachyzoites were immunolabelled with TgSUB1 AE653 antiserum and the mouse anti-SAG1 DG52 monoclonal antibody without permeabilization. Scale bar is $5 \mu \mathrm{m}$.

C. Western blot analysis of the MIC2, MIC4 and M2AP cellular and secreted products collected from the $\triangle$ sub1::FLSUB1, $\triangle$ sub1:::AGPISUB1 and $\triangle$ sub1pCAT strains. For MIC2 some cellular MIC2 is evident in addition to secreted MIC2 in the ESA from the two complemented lines (migrating just above $100 \mathrm{kDa}$ ). Blots were probed with appropriate antibodies as described in Experimental procedures. Molecular weights are in $\mathrm{kDa}$. M2APp: precursor form; M2APm: mature form. 
A
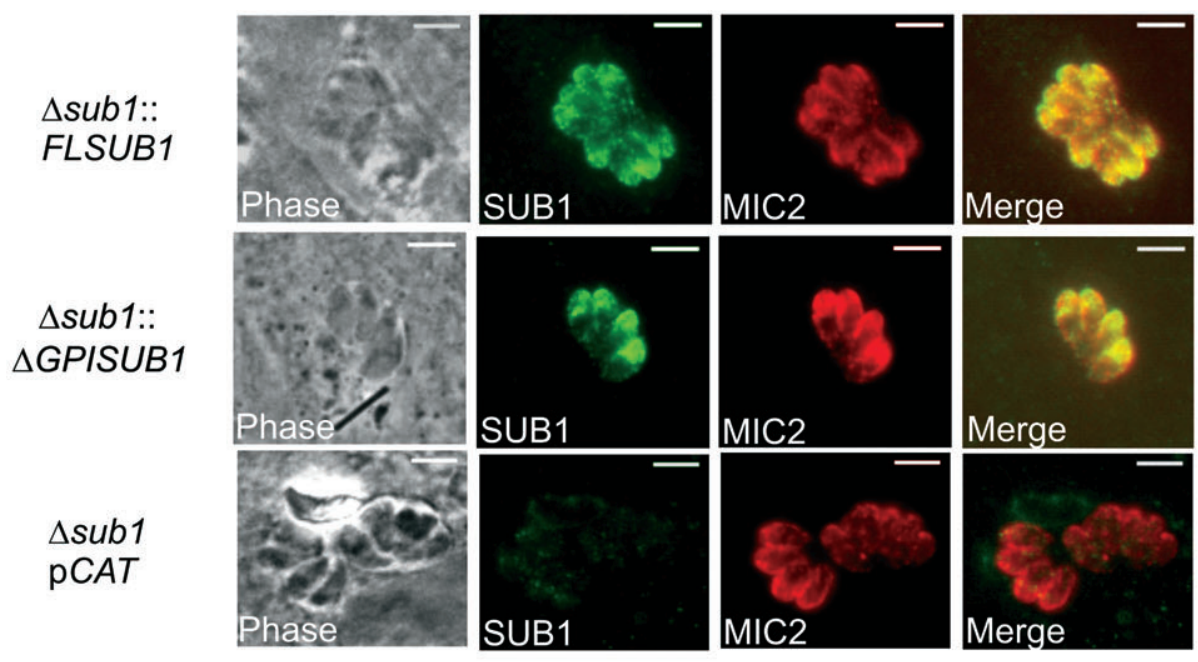

Extracellular

Invading

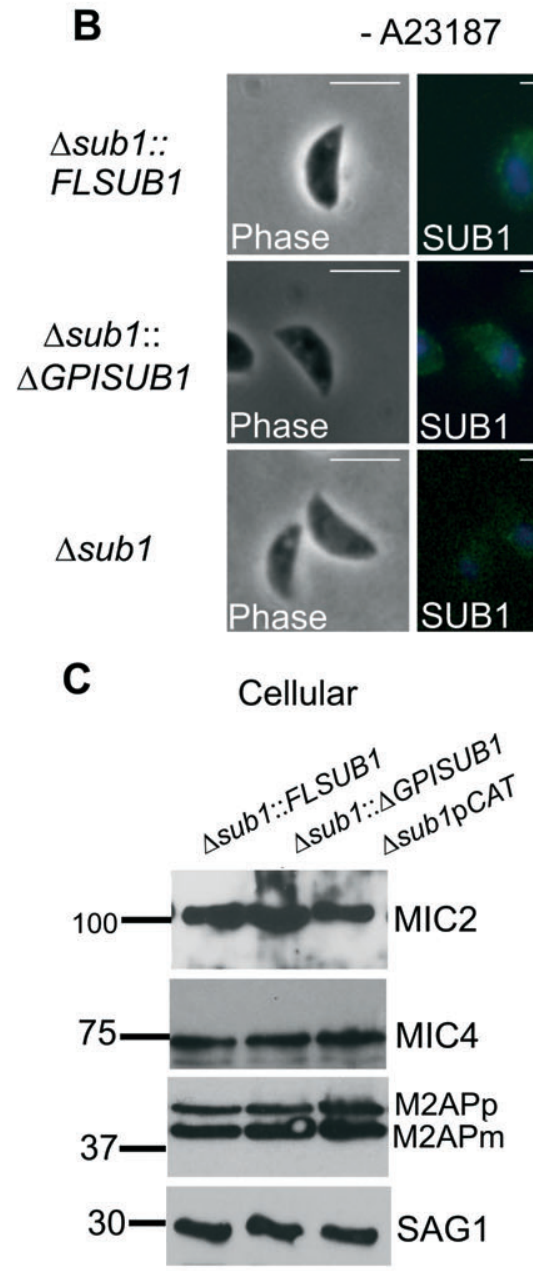

+ A23187
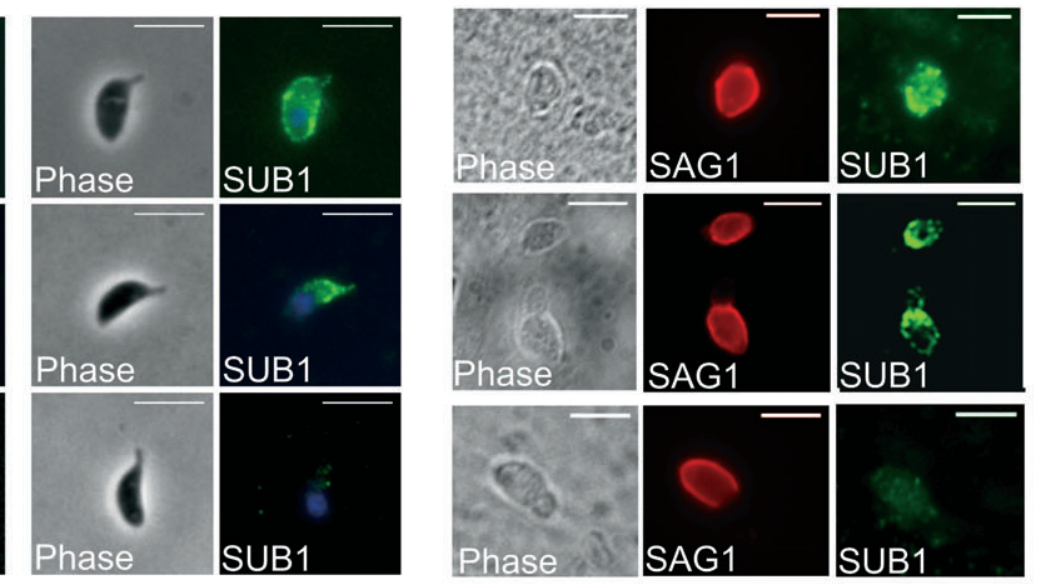

Secreted

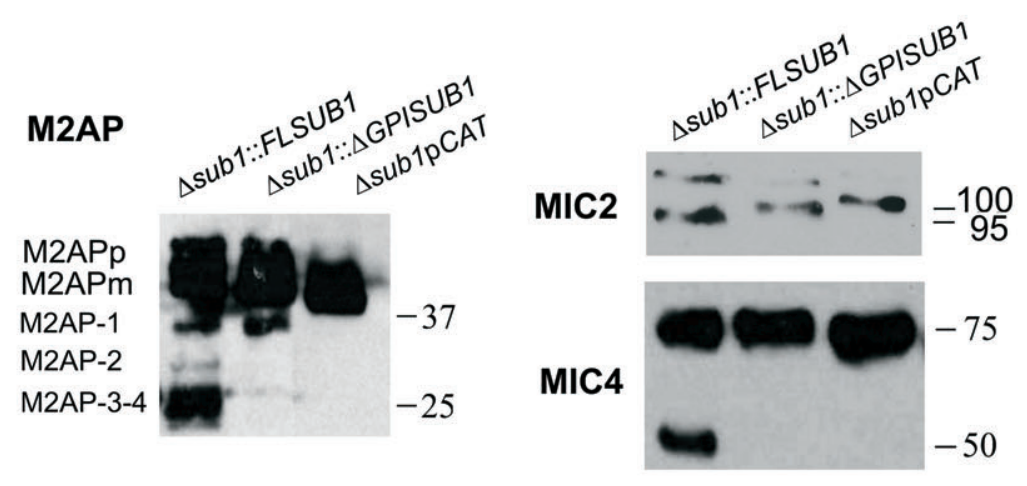

Complementation with the full-length TgSUB1 gene increased invasion ( $\Delta$ sub1::FLSUB1), but did not completely restore entry to parental levels, a finding that has been seen in several previous studies (Huynh et al., 2003;
Cerede et al., 2005). When $\Delta s u b 1$ parasites were allowed to invade for $1 \mathrm{~h}$, they showed comparable invasion to control parasites (data not shown). This suggests that $\Delta s u b 1$ parasites have a delay in invasion, a phenomenon 
A

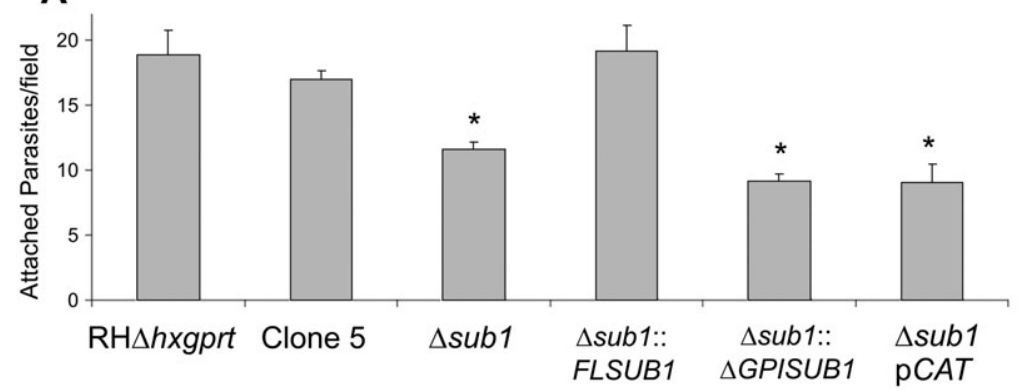

B

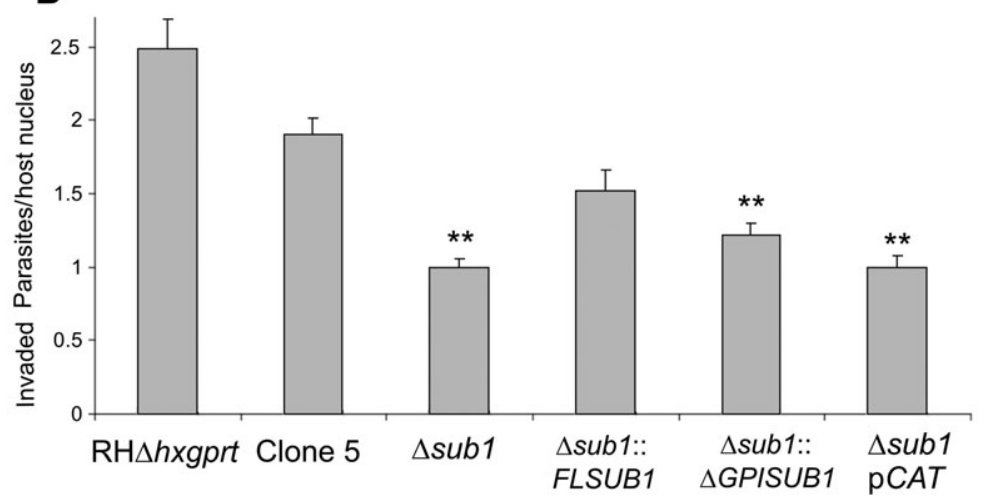

Fig. 4. Attachment and invasion phenotypes of strains lacking TgSUB1-dependent MIC cell surface processing.

A. Quantification of attachment to glutaraldehyde-fixed HFF cells expressed as the number of attached parasites counted per field. An asterisk indicates a statistically significant difference compared with $\mathrm{RH} \Delta h x g$ prt, Clone 5 and $\Delta$ sub1::FLSUB1 $(P<0.05$, two-tailed Student's $t$-test). B. Quantification of $15 \mathrm{~min}$ invasion assays expressed in number of invaded parasites per host cell counted. A double asterisk indicates a statistically significant difference compared with $\mathrm{RH} \Delta h x$ gprt and Clone $5(P<0.05$, two-tailed Student's $t$-test). Data are mean values \pm SEM. previously seen for M2AP-deficient parasites (Huynh et al., 2003).

\section{Strains lacking TgSUB1 have a defect in gliding motility}

To determine if loss of TgSUB1-dependent MIC surface processing affects other parasite processes, we analysed parasite gliding motility using a commonly used in vitro assay that assesses trail formation and gliding motility on FBS-coated coverslips. Toxoplasma motility is characterized by three forms: helical, circular and twirling. Helical motility deposits wide-arcing or straight, long trails (noncircular) in contrast to circular motility (Hakansson et al., 1999). No trails were detected for $\triangle$ sub1 and $\triangle$ sub1pCAT whereas trails were seen for $\triangle$ sub1::FLSUB1, Clone 5 and $\mathrm{RH} \Delta h x g p r t$, strains that have intact TgSUB1dependent MIC surface processing (Fig. 5A). Very short preliminary trails were detected for $\triangle$ sub1:: $\triangle$ GPISUB1 suggestive of transient gliding movements. Similar results were obtained using long incubation times (data not shown). Parasite gliding was also examined by live video microscopy. Quantification of the different forms of gliding revealed that a greater proportion of the $\Delta$ sub1 parasites were inactive compared with the $\triangle$ sub1::FLSUB1 strain with significantly less helical movement (Table 2), which could account for the lack of trail deposition.

Since the defect in gliding on FBS-coated slides was more pronounced than the defect observed in invasion, we analysed trail formation on other biologically relevant substrates including heparin, chondroitin sulfate A and collagen I with the three representative strains: $\Delta$ sub1::FLSUB1, $\triangle$ sub1:: $\triangle$ GPISUB1 and $\triangle$ sub1. Although trails could be detected in all strains with all substrates, fewer and shorter trails were detected for the $\triangle$ sub1:: $\triangle$ GPISUB1 and $\triangle$ sub1 strains when gliding on heparin and chondroitin sulfate A (Fig. 5B). To further analyse the gliding behaviour, the proportion of long trails was determined and trail length was measured (Table 3). Long trails were more frequent for the $\triangle$ sub1::FLSUB1 strain when gliding on heparin or chondroitin sulfate $A$ compared with the $\triangle$ sub1::GPISUB1 and the $\triangle$ sub1 strains. Moreover, there was a significant quantitative difference in gliding distance between the $\triangle$ sub1::FLSUB1 versus the $\triangle$ sub1:: $\triangle$ GPISUB1 and $\triangle$ sub1 strains on heparin and chondroitin sulfate $A$. Long trail per cent and gliding distance were slightly reduced on collagen I for the $\triangle$ sub1:: $\triangle$ GPISUB1 and $\triangle$ sub1 strains compared with the $\triangle$ sub1::FLSUB1; however, the differences were not statistically significant.

Live video microscopy of parasites gliding on collagen I was used to quantify the different forms of gliding (Table 2). $\Delta$ sub1 parasites showed greater activity on collagen I than on FBS, with a statistically significant increase in helical motility, explaining the recovery of trails. The videos revealed that slightly more $\triangle$ sub $1:: F L S U B 1$ parasites were active on collagen I compared with $\triangle$ sub1:: $\triangle$ GPISUB1 parasites but the per cent of helical movement were similar, correlating with static 


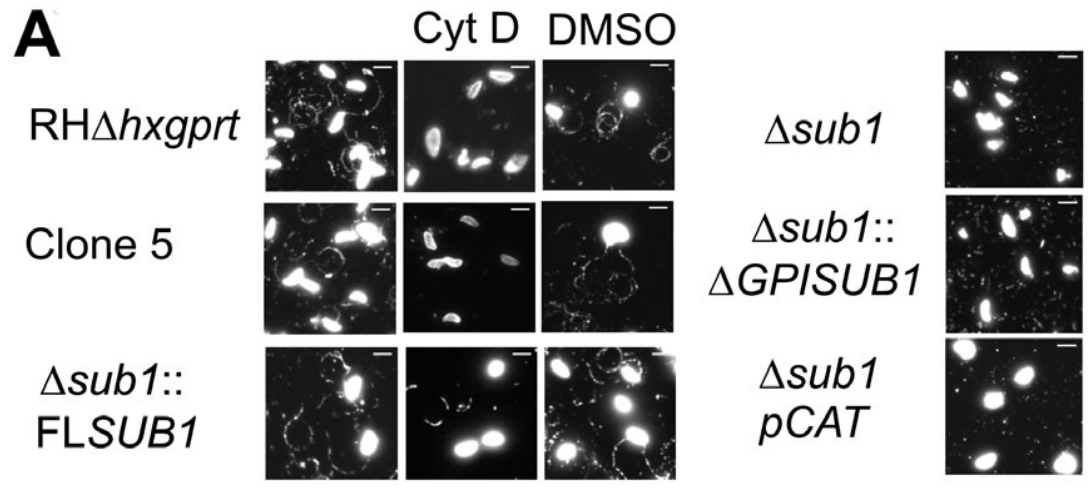

Fig. 5. Motility of strains lacking TgSUB1 activity assessed by trail deposition. All strains were allowed to glide on glass coverslips coated with FBS $(A)$ or heparin, chondroitin sulfate $A$ and collagen I (B). Trails deposited by gliding parasites were revealed with the mouse anti-SAG1 monoclonal antibody DG52 and visualized by immunofluorescence microscopy. Parasites were also pre-treated with the gliding inhibitor cytochalasin D (cytD) as negative control or with DMSO (solvent control). Scale bar is $5 \mu \mathrm{m}$.

B

$\Delta s u b 1::$ FLSUB1

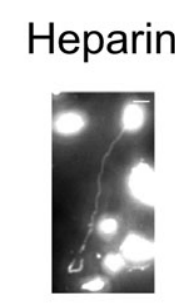

$\Delta s u b 1::$
$\triangle G P I S U B 1$
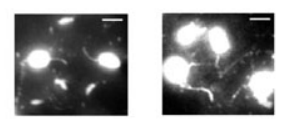

\section{Chondroitin Sulfate A}

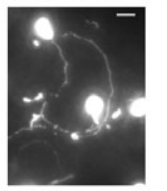

\section{Collagen I}
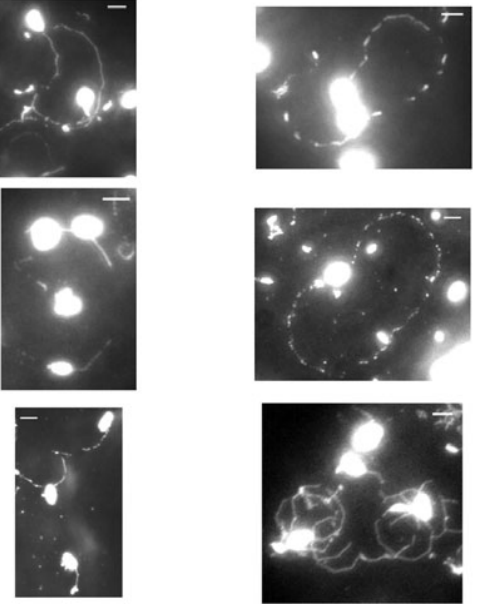

Table 2. Quantification of movement type of live gliding parasites on FBS or collagen I.

\begin{tabular}{|c|c|c|c|c|c|}
\hline $\begin{array}{l}\text { Parasite strain } \\
\text { (on FBS) } \\
\text { (on Collagen) }\end{array}$ & $\begin{array}{l}\text { Helical } \\
\% \text { FBS } \\
\% \text { Collagen }\end{array}$ & $\begin{array}{l}\text { Twirling } \\
\% \text { FBS } \\
\% \text { Collagen }\end{array}$ & $\begin{array}{l}\text { Circular } \\
\% \text { FBS } \\
\% \text { Collagen }\end{array}$ & $\begin{array}{l}\text { Inactive } \\
\% \text { FBS } \\
\% \text { Collagen }\end{array}$ & $\begin{array}{l}\text { Non-productive } \\
\% \text { FBS } \\
\% \text { Collagen }\end{array}$ \\
\hline \multicolumn{6}{|l|}{$\Delta s u b 1:: F L S U B 1$} \\
\hline (211 parasites) & $33.2 \%$ & $17.1 \%$ & $14.7 \%$ & $20.0 \%$ & $15.2 \%$ \\
\hline (128 parasites) & $38.2 \%$ & $21.9 \%$ & $3.1 \%$ & $23.4 \%$ & $13.3 \%$ \\
\hline \multicolumn{6}{|l|}{$\Delta s u b 1$} \\
\hline (190 parasites) & $4.7 \%$ & $15.3 \%$ & $8.9 \%$ & $54.0 \%$ & $17.4 \%$ \\
\hline (181 parasites) & $23.2 \%$ & $19.3 \%$ & $1.1 \%$ & $40.8 \%$ & $15.5 \%$ \\
\hline \multicolumn{6}{|c|}{$\triangle s u b 1:: \triangle G P I S U B 1$} \\
\hline (205 parasites) & ND & ND & ND & ND & ND \\
\hline (180 parasites) & $42.7 \%$ & $9.4 \%$ & $1.7 \%$ & $32.2 \%$ & $13.9 \%$ \\
\hline
\end{tabular}

Live parasites of the indicated strain were allowed to glide on substrate-coated dishes and gliding modalities were enumerated by observing individual parasites as described in Experimental procedures. The different forms of motility of the $\triangle$ sub1:: $\Delta$ GPISUB1 strain could not be reliably assessed on FBS since, rather than actively gliding on the substrate, the parasites frequently moved in the direction of the media flow of the heating element used during the video capture. Parasites that are actively gliding can go against the direction of this flow, and thus $\triangle$ sub1::FLSUB1 on FBS and collagen, and the $\triangle$ sub1 and $\triangle$ sub1:: $\triangle$ GPISUB1 on collagen (and even the $\Delta$ sub1 on FBS, those few that did glide) could be scored. The $\triangle$ sub1:: $\triangle$ GPISUB1 on FBS moved mostly in one direction, although they were able to glide circularly against the flow. The weak adhesion of the $\triangle$ sub1:: $\triangle$ GPISUB1 parasites may make them more susceptible to transient attachment and displacement in media compared with Dsub1::FLSUB1 parasites, complicating enumeration of the different gliding modes. ND, not determined. 
Table 3. Gliding behaviour of $\Delta s u b 1$ strains varies with the biological substrate.

\begin{tabular}{|c|c|c|c|c|c|c|}
\hline & \multicolumn{2}{|c|}{$\Delta$ sub1::FLSUB1 } & \multicolumn{2}{|c|}{$\Delta$ sub1:: $\triangle$ GPISUB1 } & \multicolumn{2}{|l|}{$\Delta s u b 1$} \\
\hline & $\%$ LT & Mean distance & $\%$ LT & Mean distance & $\%$ LT & Mean distance \\
\hline Heparin & $26.4 \pm 3.4$ & $4.3 \pm 0.4$ & $11.2 \pm 2.0$ & $2.7 \pm 0.4$ & $11.2 \pm 3.8$ & $2.9 \pm 0.2$ \\
\hline Chondroitin sulfate A & $29.8 \pm 6.5$ & $4.4 \pm 0.5$ & $5.2 \pm 1.0$ & $2.2 \pm 0.2$ & $12.3 \pm 5.0$ & $2.9 \pm 0.4$ \\
\hline Collagen I & $27.0 \pm 4.2$ & $4.0 \pm 0.3$ & $22.9 \pm 5.9$ & $3.2 \pm 0.2$ & $19.9 \pm 5.7$ & $3.4 \pm 0.6$ \\
\hline
\end{tabular}

Gliding behaviour of $\Delta s u b 1:: F L S U B 1, \Delta s u b 1:: \Delta G P I S U B 1$ and $\Delta s u b 1$ strains was determined using a static gliding assay on substrate-coated glass slides. Mean distances are expressed in number of parasite body length (Barragan and Sibley, 2002). We denoted a trail as long (LT) when the distance is more than five times the parasite body length. Statistically significant differences in long trail proportion and trail distance on heparin and chondroitin sulfate A were observed between the $\triangle$ sub1::FLSUB1 compared with $\Delta$ sub1:: $\triangle G P I S U B 1$ and $\Delta$ sub1 strains $(P<0.05$, two-tailed Student's $t$-test). Data represent three independent experiments.

gliding data. As gliding motility is also important for calcium-dependent induced egress (Meissner et al., $2002 b$ ), we next determined the proportion of lysed vacuoles after inducing egress with calcium ionophore. No significant differences were observed in the proportion of lysed vacuoles between the strains (data not shown).

\section{TgSUB1 expression is required for maximal virulence in the mouse model}

We tested the virulence of the different strains in vivo in the mouse model. We first tested the intraperitoneal route of infection, which is the most common method used to study in vivo virulence of type I non-cyst-forming strains. There was no significant difference in the time of death between the strains using a range of inocula (10-1000 parasites, data not shown).

Because dissemination through the vasculature is important during natural T.gondii infection, we next infected the mice intravenously. Mice infected with 250 Clone 5 or $\triangle$ sub1::FLSUB1 parasites died within 10 days. Mice infected with the $\Delta s u b 1$ and $\triangle$ sub1pCAT strains died within 14 and 15 days. Unexpectedly, the $\triangle$ sub1:: $\triangle$ GPISUB1 strain was more attenuated in intravenous infection with $15 \%$ of the mice surviving infection (Fig. 6A). Similar results were obtained with the second $\triangle$ sub1:: $\triangle$ GPISUB1 clone (17\% survival with 250 parasite inoculum, Fig. 6A). Infected mice exhibited clinical evidence of cerebral infection (palsy, atrophy, agitation), and all mice that survived infection were seropositive.

Inappropriate release of the active TgSUB1 into the blood and host tissues could activate the endothelium and induce a more potent inflammatory response compared with the $\Delta s u b 1$ strain. We therefore quantified parasite burden and host cytokine profiles after intravenous infection with $\triangle$ sub1:: $\triangle$ GPISUB1, $\Delta$ sub1 and Clone 5. $\triangle$ sub1:: $\triangle$ GPISUB1 parasite burden was diminished in all organs although this difference was statistically significant in the lungs only (Fig. 6B). Cytokine profiles elicited by the three strains differed somewhat, but were not suggestive of a more robust inflammatory response to $\triangle$ sub1:: $\triangle$ GPISUB1 parasites (Fig. 6C). These studies are consistent with a defect in either in vivo replication or dissemination for strains lacking full TgSUB1 activity, but more detailed characterization of parasite in vivo viability and host response are required.

\section{Discussion}

Cell-cell attachment is a key factor regulating diverse biological processes from immune cell migration, tumour cell spread to pathogen cell invasion. Attachment involves complex molecular interactions controlled by the

Fig. 6. Strains lacking TgSUB1 have decreased virulence in the mouse model. Two hundred and fifty tachyzoites were injected intravenously into groups of five female CD-1 mice.

A. Mouse survival was monitored daily over a period of 8 weeks. The $\Delta s u b 1$ and the $\Delta s u b 1 p C A T$ strains are less virulent than the Clone 5 and the $\triangle$ sub1::FLSUB1 strains $(P<0.05)$. The $\triangle$ sub1:: $\triangle G P I S U B 1$ was significantly less virulent than the $\triangle$ sub1::FLSUB1 and $\triangle$ sub1 strains $(P<0.001)$. Similar results were obtained with a second independent $\Delta$ sub1:: $\Delta G P I S U B 1$ clone $(\Delta s u b 1:: \Delta G P I S U B \# 2)$. Data are pooled from three independent experiments (at least 15 mice total).

B. Parasite tissue burden after 6 days of infection with the Clone $5, \Delta$ sub1, $\Delta$ sub1::FLSUB1 and $\Delta$ sub1:: $\Delta$ GPISUB1 strains. Spleen and lung parasite burdens were lower for $\triangle S$ Sub1:: $\triangle$ GPISUB1 compared with Clone $5, \Delta s u b 1$ and $\Delta s u b 1:: F L S U B 1$ strains, but data were statistically significant for the lung only (two-tailed Student's $t$-test, $P<0.05$ ). No significant differences were observed in the brain where fewer parasites were detected for all strains. Data are pooled from two independent experiments and show burdens for each mouse tested with average value represented by the dark line.

C. Mice cytokine tissue levels after 3 and 6 days of infection with Clone 5 (black bars), $\Delta$ sub1:: $\Delta$ GPISUB1 (white bars) and $\Delta$ sub1 (grey bars) strains. Significant differences in the cytokine levels were observed at day 3: spleen: for TNF $\alpha$ between the Clone 5 strain compared with the $\Delta s u b 1$ and $\Delta s u b 1:: \Delta$ GPISUB1 strains; lung: for IL12 and IL4 cytokines between the three strains and for IL10 between the $\Delta$ sub1:: $\triangle$ GPISUB1 strain compared with the Clone 5 and $\Delta$ sub1 strains. At day 6 , no significant differences in the cytokine levels were observed except for TNF $\alpha$ between the $\Delta$ sub1:: $\triangle$ GPISUB1 strain compared with the $\Delta$ sub1 and Clone 5 strains (two-tailed Student's $t$-test, $P<0.05$ ). 


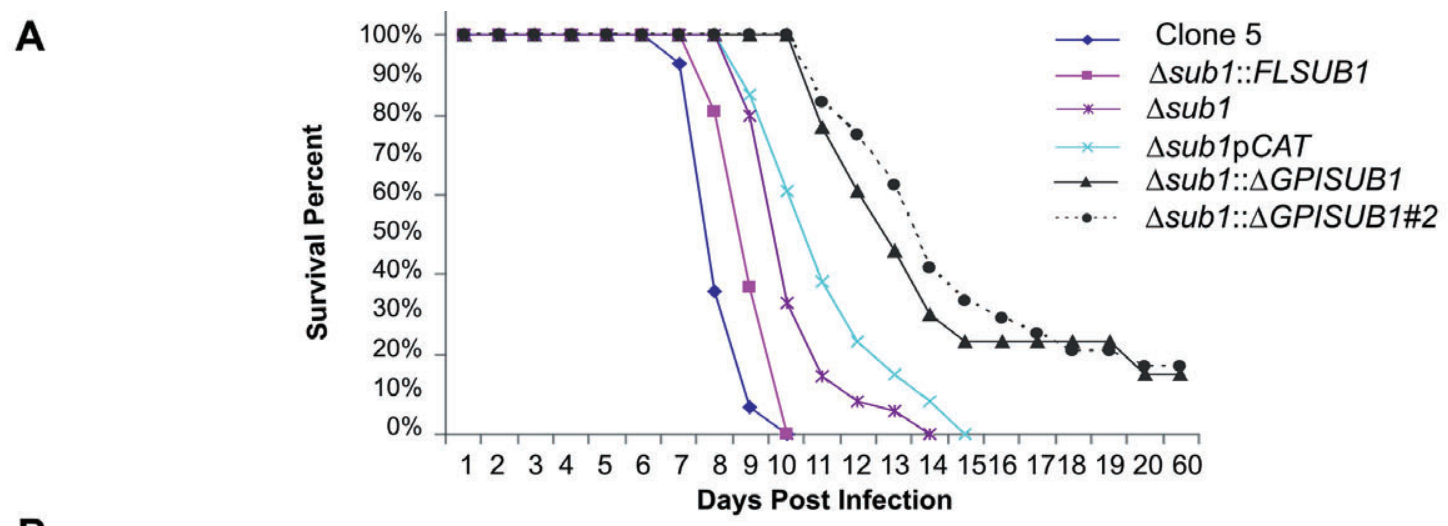

B
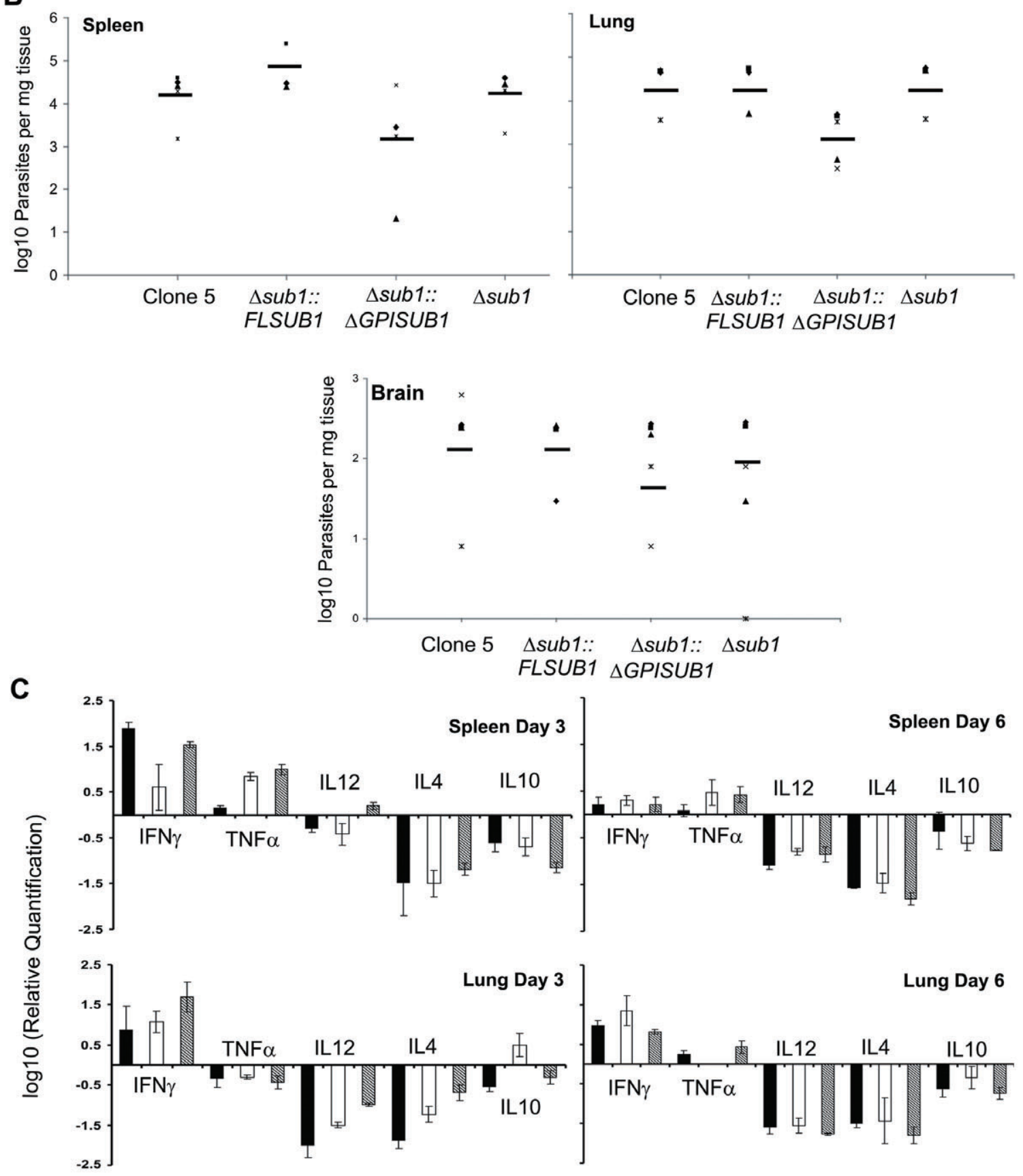
co-ordinated expression and multimerization of various adhesive receptors. Frequently the maturation and inactivation of adhesive complexes is controlled by regulated proteolysis. Migratory cells use both adhesion receptors and proteolytic enzymes to regulate their interaction with and response to extracellular matrices (Stefanidakis and Koivunen, 2006).

Attachment is a prerequisite for host cell invasion by T. gondii. Co-ordinated regulated processing of micronemal surface adhesive complexes is likely to play an important role in the host cell invasion process (Dowse and Soldati, 2004; Carruthers and Blackman, 2005; Carruthers, 2006). Microneme proteins (MIC) first undergo a surface proteolytic trimming that has been proposed to activate adhesive domains and promote interaction with host cell receptors. Although study of these processing events has been an area of extensive investigation, the precise role of MIC surface processing was previously unknown and the responsible proteases not identified.

TgSUB1 is found in the micronemes and is exposed on the parasite surface upon microneme secretion (Binder et al., 2008). $\Delta s u b 1$ parasites have a global perturbation of microneme protein processing on the parasite surface with loss of the M2AP, MIC2 and MIC4 processed fragments ascribed to MPP2 activity. Western blot and 2D-DIGE analyses revealed that the M2AP-1, the product generated by the MPP3 protease (Zhou et al., 2004), is absent in $\Delta s u b 1$ parasites. These data indicate that TgSUB1 is required for both MPP2 and MPP3 activities.

Surprisingly, only the M2AP-1 fragment is restored in the $\triangle s u b 1:: \triangle G P I S U B 1$ but processing of M2AP ascribed to MPP2 was not restored. MPP2 and MPP3 have been distinguished by their differential susceptibility to protease inhibitor ALLN, which blocks MPP2 activity but not MPP3 activity (Zhou et al., 2004). This differential inhibitor susceptibility was the basis for proposing two independent proteases termed MPP2 and MPP3.

We propose an alternative mechanism that reconciles these seemingly conflicting observations to explain how TgSUB1 could be both MPP2 and MPP3. TgSUB1 surface anchorage by GPI is unlikely to affect substrate specificity, but would influence the duration of TgSUB1 association with the cell surface and any microneme protein complexes associated with the cell surface. Microneme protein complexes and TgSUB1 are cleaved upon release onto the cell surface by MPP2 as part of adhesin inactivation and shedding. This proteolysis is enhanced by cytochalasin D treatment (see Fig. S2), which prevents redistribution of the microneme protein complexes towards the posterior end of the parasite, trapping MPP2 and its substrates together (Zhou et al., 2004). In contrast, lack of the GPI in $\Delta$ sub1:: $\Delta$ GPISUB1 would decrease the duration of interaction of TgSUB1 and its substrates at the cell surface, potentially decreasing the efficiency of proteolytic cleavage. However, the autocatalytic processing of TgSUB1 is similar whether or not the complemented allele contains a GPI (Fig. S1E).

We have previously shown that maturation and activation of TgSUB1 (Miller et al., 2001) is not blocked by protease inhibitors. In typical subtilases, this resistance to protease inhibitors is due to inability of the inhibitor to access the active site to prevent proteolytic cleavage.

Similarly, the first steps of microneme complex cleavage by TgSUB1 may not be completely inhibited by ALLN, even if TgSUB1 activity is ALLN sensitive. Prior to activation of microneme secretion, the activity of TgSUB1 will be inhibited by bound prodomain and inaccessible to protease inhibitors such as ALLN. Once microneme secretion is activated by calcium and the TgSUB1 prodomain is released, substrates and ALLN will compete to occupy the TgSUB1 active site. Since TgSUB1 will be rapidly activated after release of the prodomain, some cleavage of substrates by TgSUB1 will take place before ALLN can fully inhibit activity. Thus the first cleavage steps mediated by TgSUB1, if they occur rapidly after activation, might appear to be ALLN-resistant and appear to be mediated MPP3 rather than MPP2.

Although our data suggest that TgSUB1 is responsible for both MPP2 and MPP3 activity, we cannot formally rule out that TgSUB1 is involved in the activation of another processing protease responsible for MIC processing. The sites of self-cleavage of TgSUB1 are similar to mapped sites of cleavage by MPP2 and MPP3 (Fig. S3). However, our attempts to test the protease activity of TgSUB1 using immunoprecipitated TgSUB1 and M2AP recombinant protein were inconclusive as TgSUB1 degrades rapidly via autoproteolysis, and we have not been able to generate active recombinant TgSUB1.

MIC2, MIC4 and M2AP are shed from the parasite cell surface normally indicating that protease activity responsible for the intramembranous cleavage is not affected in the $\Delta s u b 1$ strain. These are also consistent with MPP1 being a rhomboid serine protease activity (Brossier et al., 2005; Dowse et al., 2005), and genetic studies are consistent with TgROM4 acting as MPP1 (Buguliskis et al., 2010).

Beyond the known substrates of MPP2 and MPP3, TgSUB1 candidate substrates include PLP1, a perforinlike protein that induces membrane disruption during parasite egress from host cells (Kafsack et al., 2009). We did not found any defect in egress suggesting that PLP1 processing by TgSUB1 is not required for PLP1 function during egress. The 2D-DIGE analysed also revealed several proteins that are not predicted to have signal peptides (Table S1). This may be an artefact of the greater cell lysis seen with the serum-free conditions used for large-scale ESA preparations needed for proteomic 
analysis (Zhou et al., 2004), and many of these proteins may not be secreted.

Gliding motility requires formation of successive tight interactions between surface adhesive complexes and the substratum followed by disengagement. Our data suggest that lack of MIC surface processing affects tight interaction with the substratum, impairing helical motility and leading to impaired or delayed motility depending on the substrate. Nascent trails could be detected in static assays for $\triangle$ sub1:: $\triangle$ GPISUB1 when gliding on FBS, heparin and chondroitin sulfate $A$. Video gliding on FBS showed that $\triangle$ sub1:: $\triangle$ GPISUB1 parasites do not move well on the substratum. We hypothesize that partial processing of MICs in the $\triangle$ sub1:: $\triangle$ GPISUB1 leads to weak parasite-substratum interactions that initiate gliding, leading to the short trails observed in the static assay, but that the interaction is not sufficiently strong to complete a helical glide.

Despite the profound alteration of proteolytic processing associated with loss of TgSUB1, $\Delta s u b 1$ parasites have a surprisingly modest phenotype in vitro and in vivo. Previous studies have shown that $T$. gondii invasion involves redundant adhesive domains with synergistic roles in parasite virulence (Cerede et al., 2005). T. gondii encodes 12 subtilase genes, so there may be functional redundancy in both microneme adhesins as well as the proteases involved in regulation of adhesin activity. Although loss of TgSUB1-dependent MIC surface processing affects parasite attachment, helical motility and invasion of the host cell, parasites without MPP2 activity are able to compensate. It appears that the resulting defect in adhesion affects efficiency of motility and initiation of parasitehost cell invasion, but does not result in a sustained defect in invasion or parasite replication ((Binder et al., 2008) and Fig. S1G).

The gliding phenotype of $\Delta s u b 1$ and $\Delta s u b 1:: \Delta$ GPISUB1 strains was rescued on collagen I. It has been shown that MIC2 binds to heparin but not collagen I (Harper et al., 2004) suggesting that other MIC complexes may participate in gliding on collagen I. A mutant strain that expresses only $2 \%$ of MIC2 protein has a severe defect in gliding motility on FBS with fewer trails that are shorter and mainly circular (Huynh and Carruthers, 2006). The phenotype of these parasites is not rescued when gliding on collagen I, indicating that MIC2 is required for gliding on collagen I, but that proteolytic trimming of the MIC2M2AP complex is not required (Fig. S4). Previous studies showed that full-length cellular form of MIC2 binds the host cell (Carruthers et al., 1999a). Moreover, the MIC2-I domain binds to heparin whether the $5 \mathrm{kDa} N$-terminal fragment is present or not (Harper et al., 2004). Collectively, the data suggest that initial MIC2 processing is not crucial for adhesion. We can hypothesize that sequential processing could modulate effective engagement with dif- ferent host cell ligands. Thus, parasites could compensate the loss of TgSUB1 dependent MICs processing either through binding with other MICs or through differential binding to ligands according to the degree of MICs processing. Unfortunately attempts to test this hypothesis were thwarted by uniformly low cellular binding of recombinant protein reagents tested. It is also possible that TgSUB1-dependent processing is more important for other molecular interactions that have not been examined in our study.

TgSUB1 contributes to maximal virulence in the mouse model. Here too, TgSUB1 may have a more critical function in a biological context that we did not examine. By analogy, the importance of knobs for Plasmodium falciparum adherence was only seen under flow conditions that mimic the blood stream (Crabb et al., 1997). MIC2 N-terminal trimming is reported to be necessary for ICAM-1 interaction and is correlated with trans-epithelial migration (Barragan et al., 2005). It is possible that TgSUB1 activity is more critical when parasites cross biological barriers such as the gut epithelium after ingestion of sporozoites or bradyzoites. Unfortunately, efforts to test transmigration ability did not yield consistent results, and the $\mathrm{RH}$ background stage does not make bradyzoites, a stage with numerous micronemes whose contents may be required for efficient invasion of gut epithelia.

Finally, recent analyses have revealed that the clone of $\mathrm{RH}$ strain parasites used in this study ( $\mathrm{RH}-\mathrm{ERP})$ has acquired enhanced ability to invade, glide and replicate that is not representative of other virulent type I strains (Khan et al., 2009). Thus it is possible that the true biological importance of TgSUB1 has been masked by the unusually efficient invasive abilities of our $\mathrm{RH}$ background strain. If so, deletion of TgSUB1 may have a greater phenotypic effect upon strains that have been more recently isolated from animal hosts.

\section{Experimental procedures}

\section{Parasite maintenance}

Toxoplasma gondii $\mathrm{RH}$ strain $\Delta$ hxgprt parasites (Donald and Roos, 1998), and mutants derived from this strain (all originally $\mathrm{RH}$-ERP), were maintained by continual passage in human foreskin fibroblast (HFF). Cells were grown in Dulbecco's modified Eagle medium (DMEM; Gibco) containing 10\% fetal bovine serum (FBS), 1\% Penicillin/Streptomycin and 1\% Glutamine. Parasites and cells were cultured in a $5 \% \mathrm{CO}_{2}$ atmosphere at $37^{\circ} \mathrm{C}$.

Generation of TgSUB1KO- and TgSUB1KO-complemented strains

The TgSUB1 gene was disrupted by homologous recombination with a construct containing the HXGPRT minigene flanked by 
three kilobases of the $5^{\prime}$ and $3^{\prime}$ sequence flanking the TgSUB1 coding region. Two strains with $T g S U B 1$ disruption were generated (RH gra1-GFP5S65T-tubcat $\Delta$ hxgprt and $\mathrm{RH} \Delta h x$ gprt background) (Binder et al., 2008). In each case, a clone that had integrated the TgSUB1 disruption cassette but continued to express TgSUB1 was chosen for parallel phenotypic analysis (Clone 2 for GFP strain and Clone 5 for non-GFP strain). Complementation with TgSUB1 was performed in the $\mathrm{RH}$ $\Delta s u b 1 \Delta h x g p r t$ background. Either the full-length TgSUB1 gene or the TgSUB1 gene lacking the sequence encoding for the 26 last amino acids containing the signal for GPI anchorage was amplified from cDNA. The PCR products were cloned into a plasmid containing three kilobases of $5^{\prime}$ and $3^{\prime}$ TgSUB1 flanking sequence by standard cloning procedures. The TgSUB1complemented strains were generated by co-transfecting the $\Delta$ sub1 strain with $100 \mu \mathrm{g}$ of Notl-linerarized TgSUB1 complementation construct and $10 \mu \mathrm{g}$ of Notl-linearized plasmid containing a tub-cat selection cassette. Stable lines were obtained by selection with mycophenolic acid/xanthine and chloramphenicol and cloned by limiting dilution. For each complementation, two different clones from independent transformations were isolated and tested.

\section{Indirect immunofluorescence microscopy}

For labelling of intracellular tachyzoites: HFF monolayers were grown on glass coverslips in 24-well plates until confluent. Freshly egressed tachyzoites were allowed to infect confluent cells for 20-24 h. Cells were fixed for $20 \mathrm{~min}$ in 3\% paraformaldehyde and permeabilized for $10 \mathrm{~min}$ in $0.2 \%$ Triton X-100 in PBS1X and blocked (PBS1X, BSA 3\%). Antibody dilutions used were: rabbit anti-TgSUB1 AE653 (1:500; Binder et al., 2008) and mouse anti-MIC2 mAb 6D10 (1:500, gift of David Sibley, Washington University School of Medicine, USA). Alexa Fluorconjugated secondary antibodies (Molecular Probes) were used at 1:2000 dilution. Coverslips were mounted on slides with the Vectashield mounting medium (Vector Laboratories) and viewed with an Olympus Digital Microscope (Albert Einstein College of Medicine Analytical Imaging Facility).

For labelling of extracellular tachyzoites: freshly lysed parasites were resuspended in Hank's balanced salt solution (HBSS),

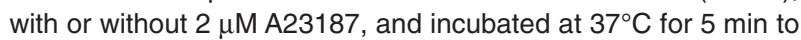
induce microneme secretion. The parasites were then added to glass coverslips before being fixed ( $3 \%$ paraformaldehyde) and blocked (PBS, BSA 3\%). Parasites were then labelled with a rabbit anti-TgSUB1 AE653 (1:500, PBS, BSA 3\%) followed by a conjugated anti-rabbit secondary antibody (Molecular probes, 1:2000). Alternatively, freshly egressed parasites were resuspended in DMEM media and allowed to infect HFF cells for 5 min at $37^{\circ} \mathrm{C}$. Invading parasites were immunolabelled with the mouse anti-SAG1 DG52 monoclonal antibody (1:500; gift from John Boothroyd, Stanford University School of Medicine) and the antiSUB1 rabbit antiserum AE653.

\section{Secretion assay}

Freshly lysed tachyzoites $\left(2-3 \times 10^{7}\right.$ parasites) were filtered, centrifuged and resuspended in DMEM 1\% FBS, 20 mM Hepes. Parasites were pre-incubated for $10 \mathrm{~min}$ at room temperature with $1 \mu \mathrm{M}$ cytochalasin $\mathrm{D}$. Microneme secretion was induced by adding $1 \%$ ethanol and warming parasites to $37^{\circ} \mathrm{C}$ for $10 \mathrm{~min}$ as previously described (Carruthers et al., 1999b). Cells were removed by centrifugation at $1000 \mathrm{~g}$ for $10 \mathrm{~min}$ at $4^{\circ} \mathrm{C}$. The supernatant containing the microneme-secreted products was used for Western blot analysis. Large-scale secretion preparations for 2D-DIGE were generated as described previously (Zhou et al., 2004).

\section{Two-dimensional differential gel electrophoresis}

Microneme-secreted contents from $1 \times 10^{9}$ parasites were analysed by 2D-DIGE as previously described (Zhou et al., 2004). Proteins were labelled using amine reactive cyanine dyes (minimal Cy dyes, GE Healthcare) according to the manufacturer's recommendations. The microneme-secreted supernatant from Clone 2 (GFP-positive) and $\triangle$ sub1GFP parasites were labelled with two different fluorochrome dyes (Cy5 or Cy3). An equal amount of the two different microneme-secreted supernatants were mixed and labelled with a third fluorochrome dye (Cy2) as an internal standard control. An equal amount of the three labelled samples were mixed together and then separated by a two-dimensional gel $(\mathrm{pH}=4.2-6.8$ first dimension and $12.5 \%$ SDS-PAGE second dimension). Spots were visualized using a Typhoon $^{\mathrm{TM}} 9400$ imager (GE Healthcare) and analysed using DeCyder-2D (v.5.0, GE Healthcare). The software analyses the overlaid signals and detects differentially expressed proteins. Proteins on the two-dimensional gel were transferred to Immobilon membrane and stained with Coomassie blue G250 and spots of interest were excised from the gel and digested with sequencing grade modified procine trypsin (Promega, http://www.promega.com). Tryptic peptides were fractionated by reverse-phase HPLC. Eluted peptides were sequenced by tandem mass spectrometry analysis. Proteins were identified by searching the data against the EPICDB Toxoplasma proteomic database (http://toro.aecom.yu.edu/biodefense; Madrid-Aliste et al., 2009) using MASCOT and are based on two or more peptide sequences with scores above the $95 \%$ confidence threshold.

\section{Western blotting}

Protein lysates were loaded on an $8 \%$ SDS gel and then transferred to nitrocellulose membrane. Membranes were probed with rabbit antisera recognizing TgSUB1 (1:10 000 PfSUB1, gift from Michael Blackman, National Institute for Medical Research, UK), SAG1-p30 (1:10 000, gift from Lloyd Kasper, Dartmouth Medical School) and MIC5 (1:10 000) followed by anti-rabbit horseradish peroxidase (HRP; 1:15 000) antibody. Secreted microneme proteins (ESA or excreted secreted antigens) were loaded on SDS gels of different resolution according to the protein probed (from $6 \%$ to $10 \%$ acrylamide). Membranes were probed with the following antibodies: mouse anti-MIC2 6D10 1:2000 (gift of David Sibley, Washington University School of Medicine, USA); rabbit anti-M2AP 1:10 000; rabbit anti-PfSUB1 1:2500) and rabbit antiMIC4 1:10 000 (gift from Dominique Soldati-Favre, University of Geneva, Switzerland). The membranes were then probed with a secondary anti-rabbit or anti-mouse antibody (1:5000-10 000) conjugated to HRP. Parasite lysis was evaluated by probing the membrane with an antibody directed against cytosolic proteins (mouse anti-actin or tubulin 1:2000). Signals were revealed with 
a peroxidase Western blot detection reagent (SuperSignal Pierce). All gels were run under reducing conditions.

\section{Quantitative real-time PCR}

RNA from freshly egressed parasites was purified using Trizol followed by chloroform extraction. Then $3.5 \mu \mathrm{g}$ of total RNA were retrotranscribed using the SuperScript kit (Invitrogen). Quantitative real-time PCR was performed on the $7300 \mathrm{ABI}$ apparatus using the Power SYBR Green Master Mix (Applied Biosystems) according to the manufacturer's instructions. PCR primers were designed with the Primer Express software (Applied Biosystems) to amplify a $100 \mathrm{bp}$ target gene fragment. PCR was performed in a $10 \mu \mathrm{l}$ volume containing $7 \mathrm{ng}$ of $\mathrm{cDNA}$ and $0.3 \mu \mathrm{M}$ of each primer. cDNA levels were normalized to cDNA $\alpha$-tubulin levels. Experiments were performed thee times with two different RNA preparations.

\section{Invasion and attachment assays}

Red/green invasion assays were performed as previously described (Huynh et al., 2003). HFF monolayer coverslips were infected with freshly egressed filtered tachyzoites $\left(7.5 \times 10^{6}\right.$ per chamber) for $15 \mathrm{~min}$ at $37^{\circ} \mathrm{C}$. The coverslips were then fixed (paraformaldehyde 3\%), and blocked (PBS1X, BSA 3\%). External attached parasites were labelled with a mouse anti-SAG1 DG52 antibody (1:500; gift from John Boothroyd, Stanford University School of Medicine) followed by a conjugated anti-mouse secondary antibody. Samples were then methanol permeabilized, blocked, and all parasites were labelled with a rabbit antiToxoplasma antiserum (Y321 1:1500; gift from Louis Weiss, Albert Einstein College of Medicine) followed by a conjugated anti-rabbit secondary antibody. Data were compiled from four independent experiments counting 10 fields per clone per coverslip at $60 \times$ magnification. The number of invaded parasites is the difference between the total number of parasites (green) and the number of attached parasites (red).

For attachment assays, $1 \times 10^{7}$ parasites were loaded with $1 \mu \mathrm{M}$ calcein-AM, $30 \mathrm{~min}$ at room temperature (Invitrogen) and then allowed to attach for $15 \mathrm{~min}$ at $37^{\circ} \mathrm{C}$ on fixed HFF cells $(2 \%$ glutaraldehyde for $5 \mathrm{~min}$ at $4^{\circ} \mathrm{C}$, blocking with $0.16 \mathrm{M}$ ethanolamine). Data were compiled from four independent experiments counting 20 fields per clone per coverslip at $40 \times$ magnification.

\section{Replication/growth rate assay}

Freshly lysed filtered parasites $\left(5 \times 10^{4}\right)$ were allowed to invade and replicate in HFF coverslip monolayers for 24 or $38 \mathrm{~h}$. Cells were fixed, permeabilized and immunolabelled with the rabbit anti-Toxoplasma antiserum Y321 (1:1500) followed by a conjugated anti-rabbit secondary antibody. The number of parasites per vacuole was enumerated on duplicate coverslips counting approximately 100 vacuoles per time point. Growth rate was determined in three independent experiments.

\section{Induced egress assay}

Egress was induced with the ionophore A23187 as previously described (Black et al., 2000). Briefly, HFF 24-well coverslip monolayers were inoculated with $10^{5}$ parasites and allowed to grow for $38 \mathrm{~h}$. Host cells were incubated for $3 \mathrm{~min}$ at $37^{\circ} \mathrm{C}$ with modified HBSS ( $1 \mathrm{mM} \mathrm{MgCl}, 1 \mathrm{mM} \mathrm{CaCl}, 10 \mathrm{mM} \mathrm{NaHCO}_{3}$, $20 \mathrm{mM}$ Hepes) containing $2 \mu \mathrm{M}$ A23187 or DMSO (control). Cells were fixed with paraformaldehyde and vacuoles were labelled by indirect immunofluorescence microscopy.

\section{Static gliding assay}

Glass coverslips were coated overnight at $4^{\circ} \mathrm{C}$ with $50 \%$ FBS/ $50 \%$ PBS1X or $50 \mu \mathrm{g} \mathrm{ml}^{-1}$ heparin, chondroitin sulfate $A$ or collagen I (solubilized in acetic acid). Freshly lysed filtered tachyzoites were resuspended in HHE (HBSS, $10 \mathrm{mM}$ Hepes, $1 \mathrm{mM}$ EGTA) and $250 \mu \mathrm{l}$ was inoculated on the coated slides for $30 \mathrm{~min}$ at $37^{\circ} \mathrm{C}$. Slides were then fixed with $3 \%$ paraformaldehyde. Trails left by gliding parasites were visualized at $40 \times$ by staining with the mouse anti-SAG1 DG52 antibody followed by a conjugated anti-mouse secondary antibody. Between 40 and 200 trails were enumerated per strain per treatment. Only trails associated with a parasite were counted. A trail was considered as circular if the diameter was approximately the length of a tachyzoite; trails larger in diameter or straight were considered as non-circular. Trail lengths were measured in parasite body length.

\section{Live gliding microscopy}

One T25 flask of parasites was filter-purified, pelleted and resuspended in $10 \mathrm{ml}$ of HHE (HBSS, $10 \mathrm{mM}$ Hepes, $1 \mathrm{mM}$ EGTA). Two millilitres of parasites was placed in a $35 \mathrm{~mm}$ glass bottom Petri dish and allowed to settle at room temperature for $10 \mathrm{~min}$, before placing on a $37^{\circ} \mathrm{C}$ heating block for $5 \mathrm{~min}$ to activate parasites. The dish was transferred to a heated ring placed on the stage of a Zeiss Axio inverted microscope. Videos were captured at one frame per second for $1 \mathrm{~min}$, using the Axio time-lapse software. The type of gliding movement was enumerated by observing individual parasites for each strain on each substrate. Maximum projection images showing the motility patterns over the $1 \mathrm{~min}$ period were generated by exporting the individual frame and reimporting them as Z-stacks. The time-lapse movies were generated by exporting the frames from the $1 \mathrm{~min}$ period, increasing the speed to $5 \times$, and converted the image series to an avi file.

\section{In vivo virulence assay}

Groups of four to five female CD-1 mice between 7 and 9 weeks of age were infected intraperitoneally or intravenously with different parasite inocula. The survival of mice after infection was monitored daily over a period of 8 weeks. Plaque assays were performed with the different inocula to ensure equal amounts of viable injected parasites. The seroconversion of all surviving mice was assayed 4 weeks post infection by Western blot analysis by probing mouse serum against $\mathrm{RH}$ tachyzoite lysate. Results represent three independent experiments. Care and handling of animals was performed in accordance with AAALAC guidelines after approval of protocols by the Albert Einstein College of Medicine Animal Use Committee. 


\section{Parasite tissue burden and cytokine tissue level} quantification

Groups of two or three female CD-1 mice between 7 and 9 weeks of age were infected intravenously with 250 parasites of the Clone 5, $\Delta$ sub1, $\Delta$ sub1::FLSUB1 and $\triangle$ sub1:: $\triangle$ GPISUB1 strains. Six days after injection, spleen, lung and brain were collected and DNA was purified using TRIzol. Parasite genomic DNA load was determined by quantitative real-time PCR using parasite specific primers (Tox 9-11; Reischl et al., 2003). Number of parasites per mg was calculated using a standard curve generated with genomic DNA from $\mathrm{RH} \Delta h x g$ prt tachyzoites. Groups of three mice were infected intravenously with 250 parasites of the Clone 5, $\triangle$ sub $1:: \Delta$ GPISUB1 and $\Delta$ sub1 strains. At days 3 and 6 after injection, RNA was purified using TRIzol. Five micrograms of total RNA was retrotranscribed using the SuperScript kit (Invitrogen) and $1 \mu \mathrm{l}$ of cDNA was used in $10 \mu \mathrm{l}$ of SYBR green quantitative real-time PCR reaction. Cytokine levels are shown as the relative proportion of cDNA compared with uninfected tissue and normalized to GAPDH cDNA levels.

\section{Statistical analysis}

Statistical analyses were performed using the Prism Graph pad software. Two-tailed Student's $t$-test was used for analysis of invasion, attachment, replication, egress and gliding assays. The level of significance for mouse virulence assays was determined with the Kaplan-Meier estimator. Differences were considered significant if $P$-value was $<0.05$.

\section{Acknowledgements}

This work was supported by NIH-NIAID R01 Al46985 (to K.K.) and RO1 Al046675 (V.B.C.). E.M.B. and P.K.H. were supported by NIH-NIAID training Grant 5T32-A107506, awarded to the Albert Einstein College of Medicine. Some of the data in this manuscript were published as part of a thesis submitted by E.M.B. in partial fulfilment of the requirements for the Degree of Doctor of Philosophy in the Sue Golding Graduate Division of Medical Sciences, Albert Einstein College of Medicine, Yeshiva University.

\section{References}

Barragan, A., and Sibley, D.L. (2002) Transepithelial migration of Toxoplasma gondii is linked to parasite motility and virulence. J Exp Med 195: 1625-1633.

Barragan, A., Brossier, F., and Sibley, D.L. (2005) Transepithelial migration of Toxoplasma gondii involves an interaction of intercellular adhesion molecule 1 (ICAM-1) with the parasite adhesin MIC2. Cell Microbiol 7: 561-568.

Baum, J., Papenfuss, A., Baum, B., Speed, T., and Cowman, A. (2006) Regulation of apicomplexan actin-based motility. Nat Rev Microbiol 4: 621-628.

Binder, E.M., Lagal, V., and Kim, K. (2008) The prodomain of Toxoplasma gondii GPI-anchored subtilase TgSUB1 mediates its targeting to micronemes. Traffic 9: 1485-1496.

Black, M., Arrizabalaga, G., and Boothroyd, J. (2000) lonophore-resistant mutants of Toxoplasma gondii reveal host cell permeabilization as an early event in egress. Mol Cell Biol 20: 9399-9408.
Blumenschein, T.M., Friedrich, N., Childs, R.A., Saouros, S., Carpenter, E.P., Campanero-Rhodes, M.A., et al. (2007) Atomic resolution insight into host cell recognition by Toxoplasma gondii. EMBO J 26: 2808-2820.

Brecht, S., Carruthers, V.B., Ferguson, P.J.D., Giddings, O.K., Wang, G., Jakle, U., et al. (2001) The toxoplasma micronemal protein MIC4 is an adhesin composed of six conserved apple domains. J Biol Chem 276: 41194127.

Brossier, F., Jewett, T.J., Lovett, J.L., and Sibley, D.L. (2003) C-terminal processing of the toxoplasma protein MIC2 is essential for invasion into host cells. J Biol Chem 278: 6229-6234.

Brossier, F., Jewett, T.J., Sibley, D.L., and Urban, S. (2005) A spatially localized rhomboid protease cleaves cell surface adhesins essential for invasion by Toxoplasma. Proc Natl Acad Sci USA 102: 4146-4151.

Brydges, S.D., Zhou, X.W., Huynh, M.H., Harper, J.M., Mital, J., Adjogble, K.D., et al. (2006) Targeted deletion of MIC5 enhances trimming proteolysis of Toxoplasma invasion proteins. Eukaryot Cell 5: 2174-2183.

Buguliskis, J.S., Brossier, F., Shuman, J., and Sibley, L.D. (2010) Rhomboid 4 (ROM4) affects the processing of surface adhesins and facilitates host cell invasion by Toxoplasma gondii. PLoS Pathog 6: e1000858.

Carruthers, V.B. (2006) Proteolysis and Toxoplasma invasion. Int J Parasitol 36: 595-600.

Carruthers, V., and Blackman, M. (2005) A new release on life: emerging concepts in proteolysis and parasite invasion. Mol Microbiol 55: 1617-1630.

Carruthers, V., and Sibley, L. (1997) Sequential protein secretion from three distinct organelles of Toxoplasma gondii accompanies invasion of human fibroblasts. Eur $J$ Cell Biol 73: 114-123.

Carruthers, V.B., Giddings, O.K., and Sibley, D.L. (1999a) Secretion of micronemal proteins is associated with toxoplasma invasion of host cells. Cell Microbiol 1: 225235.

Carruthers, V., Moreno, S., and Sibley, L. (1999b) Ethanol and acetaldehyde elevate intracellular $\left[\mathrm{Ca}^{2+}\right]$ and stimulate microneme discharge in Toxoplasma gondii. Biochem $J$ 342: 379-386.

Carruthers, V.B., Sherman, G.B., and Sibley, D.L. (2000) The Toxoplasma adhesive protein MIC2 is proteolytically processed at multiple sites by two parasite-derived proteases. J Biol Chem 275: 14346-14353.

Cerede, O., Dubremetz, J., Bout, D., and Lebrun, M. (2002) The Toxoplasma gondii protein MIC3 requires pro-peptide cleavage and dimerization to function as adhesin. EMBO 21: 2526-2536.

Cerede, O., Dubremetz, J., Soete, M., Deslee, D., Vial, H., Bout, D., and Lebrun, M. (2005) Synergistic role of micronemal proteins in Toxoplasma gondii virulence. J Exp Med 201: 453-463.

Cesbron-Delauw, M.F., Guy, B., Torpier, G., Pierce, R.J., Lenzen, G., Cesbron, J.Y., et al. (1989) Molecular characterization of a 23-kilodalton major antigen secreted by Toxoplasma gondii. Proc Natl Acad Sci USA 86: 75377541.

Conseil, V., Soete, M., and Dubremetz, J. (1999) Serine protease inhibitors block invasion of host cells by Toxo- 
plasma gondii. Antimicrob Agents Chemother 43: 13581361.

Crabb, B., Cooke, B., Reeder, J., Waller, R., Caruana, S., Davern, K., et al. (1997) Targeted gene disruption shows that knobs enable malaria-infected red cells to cytoadhere under physiological shear stress. Cell 89: 287-296.

Dobrowolski, J., and Sibley, L. (1996) Toxoplasma invasion of mammalian cells is powered by the actin cytoskeleton of the parasite. Cell 84: 933-939.

Donald, R.G.K., and Roos, D.S. (1998) Gene knock-outs and allelic replacements in Toxoplasma gondii: HXGPRT as a selectable marker for hit and run mutagenesis. Mol Biochem Parasitol 91: 295-305.

Dowse, T.J., and Soldati, D. (2004) Host cell invasion by the apicomplexans: the significance of microneme protein proteolysis. Curr Opin Microbiol 7: 388-396.

Dowse, T.J., Pascall, J.C., Brown, K.D., and Soldati, D. (2005) Apicomplexa rhomboids have a potential role in microneme protein cleavage during host cell invasion. Int $J$ Parasitol 35: 747-756.

Dubremetz, J., Garcia-Réguet, N., Conseil, V., and Fourmaux, M. (1998) Apical organelles and host-cell invasion by Apicomplexa. Int J Parasitol 28: 1007-1013.

Fourmaux, M., Achbarou, A., Mercereau-Puijalon, O., Biderre, C., Briche, I., Loyens, A., et al. (1996) The MIC1 microneme protein of Toxoplasma gondii contains a duplicated receptor-like domain and binds to host cell surface. Mol Biochem Parasitol 83: 201-210.

Friedrich, N., Santos, J.M., Liu, Y., Palma, A.S., Leon, E., Saouros, S., et al. (2010) Members of a novel protein family containing microneme adhesive repeat domains act as sialic acid-binding lectins during host cell invasion by apicomplexan parasites. J Biol Chem 285: 20642076.

Garcia-Réguet, N., Lebrun, M., Fourmaux, M., MercereauPuijalon, O., Mann, T., Beckers, C., et al. (2000) The microneme protein MIC3 of Toxoplasma gondii is a secretory adhesin that binds to both the surface of the host cells and the surface of the parasite. Cell 2: 353-364.

Hakansson, S., Morisaki, H., Heuser, J., and Sibley, L.D. (1999) Time-lapse video microscopy of gliding motility in Toxoplasma gondii reveals a novel, biphasic mechanism of cell locomotion. Mol Biol Cell 10: 3539-3547.

Harper, J., Hoff, E., and Carruthers, V. (2004) Multimerization of the Toxoplasma gondii MIC2 integrin-like A-domain is required for binding to heparin and human cells. Mol Biochem Parasitol 134: 201-212.

Harper, J., Huynh, M., Coppens, I., Parussini, F., Moreno, S., and Carruthers, V.B. (2006) A cleavable propeptide influences Toxoplasma infection by facilitating the trafficking and secretion of the TgMIC2-M2AP invasion complex. Mol Biol Cell 17: 4551-4563.

Huynh, M.-H., and Carruthers, V.B. (2006) Toxoplasma MIC2 is a major determinant of invasion and virulence. PLoS Pathog 2: e84. doi:10.1371/journal.ppat.0020084

Huynh, M., Rabenau, K., Harper, J., Beatty, W., Sibley, L., and Carruthers, V. (2003) Rapid invasion of host cells by Toxoplasma requires secretion of the MIC2-M2AP adhesive protein complex. EMBO J 22: 2082-2090.

Jewett, T.J., and Sibley, L.D. (2004) The toxoplasma proteins MIC2 and M2AP form a hexameric complex necessary for intracellular survival. $J$ Biol Chem 279: 9362-9369.

Kafsack, B.F., Pena, J.D., Coppens, I., Ravindran, S., Boothroyd, J.C., and Carruthers, V.B. (2009) Rapid membrane disruption by a perforin-like protein facilitates parasite exit from host cells. Science 323: 530-533.

Keeley, A., and Soldati, D. (2004) The glideosome: a molecular machine powering motility and host-cell invasion by Apicomplexa. Trends Cell Biol 14: 528-532.

Khan, A., Behnke, M.S., Dunay, I.R., White, M.W., and Sibley, L.D. (2009) Phenotypic and gene expression changes among clonal type I strains of Toxoplasma gondii. Eukaryot Cell 8: 1828-1836.

Madrid-Aliste, C.J., Dybas, J.M., Angeletti, R.H., Weiss, L.M., Kim, K., Simon, I., and Fiser, A. (2009) EPIC-DB: a proteomics database for studying Apicomplexan organisms. BMC Genomics 10: 38.

Meissner, M., Reiss, M., Viebig, N., Carruthers, V.B., Toursel, C., Tomavo, S., et al. (2002a) A family of transmembrane microneme proteins of Toxoplasma gondii contain EGF-like domains and function as escorters. J Cell Sci 115: 563574.

Meissner, M., Schluter, D., and Soldati, D. (2002b) Role of Toxoplasma gondii myosin A in powering parasite gliding and host cell invasion. Science 298: 837-840.

Miller, S.A., Binder, E.M., Blackman, M.J., Carruthers, V.B., and Kim, K. (2001) A conserved subtilisin-like protein TgSUB1 in microneme organelles of Toxoplasma gondii. J Biol Chem 276: 45341-45348.

Rabenau, K.E., Sohrabi, A., Tripathy, A., Reitter, C., Ajioka, J.W., Tomley, F.M., and Carruthers, V.B. (2001) TgM2AP participates in Toxoplasma gondii invasion of host cells and is tightly associated with the adhesive protein TgMIC2. Mol Microbiol 41: 537-547.

Reischl, U., Bretagne, S., Kruger, D., Ernault, P., and Costa, J.M. (2003) Comparison of two DNA targets for the diagnosis of Toxoplasmosis by real-time PCR using fluorescence resonance energy transfer hybridization probes. BMC Infect Dis 3: 7.

Reiss, M., Viebig, N., Brecht, S., Fourmaux, M., Soete, M., Di Cristina, M., et al. (2001) Identification and characterization of an escorter for two secretory adhesins in Toxoplasma gondii. J Cell Biol 152: 563-578.

Saouros, S., Edwards-Jones, B., Reiss, M., Sawmynaden, K., Cota, E., Simpson, P., et al. (2005) A novel galectin-like domain from Toxoplasma gondii micronemal protein 1 assists the folding, assembly, and transport of a cell adhesion complex. J Biol Chem 280: 38583-38591.

Stefanidakis, M., and Koivunen, E. (2006) Cell-surface association between matrix metalloproteinases and integrins: role of the complexes in leukocyte migration and cancer progression. Blood 108: 1441-1450.

Teo, C., Zhou, X., Bogyo, M., and Carruthers, V. (2007) Cysteine protease inhibitors block Toxoplasma gondii microneme secretion and cell invasion. Antimicrob Agents Chemother 51: 679-688.

Zhou, X.W., Blackman, M.J., Howell, S.A., and Carruthers, V.B. (2004) Proteomic analysis of cleavage events reveals a dynamic two-step mechanism for proteolysis of a key parasite adhesive complex. Mol Cell Proteomics 3: 565576. 


\section{Supporting information}

Additional Supporting Information may be found in the online version of this article:

Fig. S1. Phenotypic analyses of TgSUB1 knock-out and complemented strains.

A. Schematic representation of knock-out and complementation strategies.

B. Southern blot analysis of Xbal-digested genomic DNA from $\mathrm{RH} \Delta h x$ gprt (1), Clone 5 (2), $\Delta$ sub1 (3), $\Delta$ sub1::FLSUB1 (4), $\triangle$ sub1:: $\triangle$ GPISUB1 (5) and $\triangle$ sub1pCAT (6) strains. Digested DNA was probed with:

- A HXGPRT probe which hybridizes with the hxgprt endogenous gene (arrow) and with the HXGPRT selection cassette. The selection cassette integrated at different loci for the Clone 5 strain and the $\Delta s u b 1$ and $\Delta s u b 1$-derived strains. For the

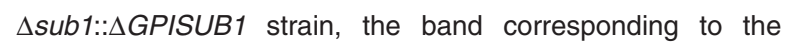
HXGPRT cassette shifted to a higher molecular weight compared with the $\Delta$ sub1 strain (close to the hxgprt endogenous gene band) consistent with complementation at the TgSUB1 locus.

- A chloramphenicol acetyl transferase (CAT) selection cassette probe which hybridizes only with the complemented strains. The selective cassette integrated at different loci in the three complemented strains (indicated by the stars).

- A probe spanning the end of the first TgSUB1 gene exon and the first intron (TgSUB1 genomic locus) which hybridizes only with the $\mathrm{RH} \Delta h x g$ prt and Clone 5 strains (arrow).

- A probe which hybridizes with the synthetic version of the TgSUB1 gene (TgSUB1 synthetic) introduced in the $\triangle$ sub1::FLSUB1 and $\triangle$ sub1:: $\triangle$ GPISUB1 strains (a weak crossreaction with the sub1 endogenous gene can be observed for the $\mathrm{RH} \Delta h x g$ prt and Clone 5 strains denoted by the star). DNA ladder sizes are indicated in $\mathrm{kb}$.

TgSUB1 expression in $\Delta s u b 1$ and the complemented strains was assessed by quantitative real-time PCR and Western blot analyses.

C. For quantitative real-time PCR, cDNA generated from RNA preparation were amplified with primers specific for Tgsub1 and cDNA levels were normalized to $\alpha$-tubulin cDNA levels using two different sets of primer. Results are shown as the relative proportion of TgSUB1 cDNA level for the different strains compared with the $\Delta s u b 1$ strain.

D and E. Western blot analysis of the TgSUB1 cellular (D) and secreted products (E) collected from the $\mathrm{RH} \Delta h \times g$ prt, Clone 5 , $\Delta$ sub1, $\Delta$ sub1::FLSUB1, $\triangle$ sub1:: $\triangle$ GPISUB1 and $\triangle$ sub1pCAT strains. Parasite lysates and secreted products were probed with the PfSUB1 antibody. MIC5 and SAG1 are used as internal controls for protein loading.

F. Western blot analysis of apical organelles content from $\Delta$ sub1complemented strains. Tachyzoite lysate blots were probed with antibodies to microneme or rhoptry proteins 1: $\triangle$ sub1::FLSUB1,

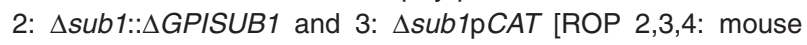
monoclonal antibody, gift from J.F. Dubremetz, University of Montpellier, France; MIC6: rabbit antisera, gift from D. SoldatiFavre, University of Geneva, Switzerland; MIC3: mouse T42F3 monoclonal antibody, gift of J.F. Dubremetz, University of Montpellier, France; which recognizes native MIC3 (Garcia-Réguet et al., 2000)]. All molecular weights are in $\mathrm{kDa}$.

G. $\Delta s u b 1$ strains have comparable rates of intracellular replication. Tachyzoites were allowed to invade and replicate in HFF cells for 24 and $38 \mathrm{~h}$. Parasites were labelled by indirect immunofluorescence with the Y321 rabbit general anti-Toxoplasma antibody (gift of L. Weiss, Albert Einstein College of Medicine). The number of parasites per vacuole was enumerated by counting 100 vacuoles at each time point. Results represent three independent experiments and are expressed as mean values \pm SEM. Fig. S2. $\Delta s u b 1$ (KO) lacks both MPP2 (ALLN-sensitive) and MPP3 (ALLN-resistant) activities. ESA preparations were made from $\mathrm{RH} \Delta h x g p r t(\mathrm{RH})$ or $\Delta s u b 1$ (knock-out or $\mathrm{KO}$ ) strain in the presence of DMSO, Cytochalasin D or ALLN. Western blots were probed with antibodies to TgSUB1, MIC4, M2AP and PLP1. For TgSUB1, processing of $90 \mathrm{kDa}$ (pro) to $80 \mathrm{kDa}$ (mat) is ALLN resistant, but other processing steps are ALLN sensitive. For MIC4, processing of the $72 \mathrm{kDa}$ (mat) form to $70 \mathrm{kDa}$ product (proc) is ALLN resistant, whereas processing to $50 \mathrm{kDa}$ fragment is ALLN sensitive. For M2AP, processing from pro M2AP (pro) to mature M2AP (mat) is present in the knock-out (thought to be performed by Cathepsin $L$ ), whereas processing from mature M2AP to M2AP-1 (largest proc form) is ALLN resistant. The other processing steps to M2AP-2, $-3,-4$ are ALLN sensitive. Cytochalasin D accelerates M2AP processing. For PLP1, processing is partially ALLN sensitive. Note that the ALLN 'resistant' processing (MPP3 activity) shows accumulation of precursor, suggesting partial inhibition of cleavage by ALLN.

Fig. S3. Alignment of putative cleavage sites. N-terminal sequencing of the MIC2 N-terminal processed form yielded sequences encompassing three putative cleavage sites. Cleavage sites for M2AP were mapped from Zhou et al. (2004). TgSUB1 prodomain autocatalytic cleavage site was identified in Miller et al. (2001). The region identified for MIC4 C-terminal processing is between amino acids 402 and 411 and encompasses six putative cleavage sites. The putative cleavage sites are situated between Apple domain pairs A3-A4 and A5-A6. Alignment of cleavage sites shows a non-specific cleavage with preference for small non-charged residues. Cleavage sites for TgSUB1 and MIC4 are largely consistent with those previously defined MPP2 and MPP3 $\left({ }^{*}\right)$ cleavage. The first N-terminal processing of MIC4 is resistant to ALLN activity (see Fig. S2). N, $\mathrm{N}$-terminal processing; C, C-terminal processing.

Fig. S4. Gliding phenotype of the MIC2 conditional knock-out strain by static assay. The MIC2 conditional knock-out strain was described in Huynh and Carruthers (2006). This strain has a tetracycline-regulated copy of mic2 introduced into the tTA background strain with disruption of the endogenous mic2 gene. Before proceeding to gliding assay, the MIC2 conditional knockout was cultured in the presence of anhydrotetracycline to deplete MIC2 expression. Gliding motility was assessed by trail deposition as described in Experimental procedures. As previously reported, diminution of MIC2 expression results in loss of non-circular gliding as shown in the figure where majority of the trail detected were circular. Gliding on collagen I did not rescue the phenotype observed on FBS. FBS: $40 \% \mathrm{NC}$ and $60 \% \mathrm{C}$; Collagen I: $46 \% \mathrm{NC}$ and $54 \% \mathrm{C}(P$-value $=0.36)$.

Table S1. Summary of the 2D-DIGE-identified proteins without a signal peptide.

Please note: Wiley-Blackwell are not responsible for the content or functionality of any supporting materials supplied by the authors. Any queries (other than missing material) should be directed to the corresponding author for the article. 